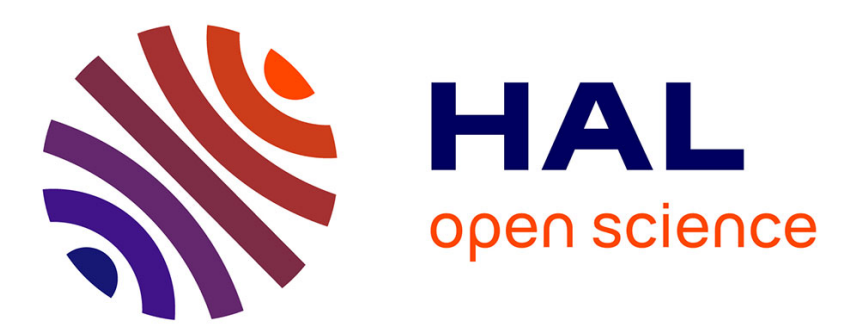

\title{
Instabilities in a cylindrical cavity heated from below with a free surface. II. Effect of a horizontal magnetic field.
}

\author{
Ridha Touihri, Anas El Gallaf, Daniel Henry, Hamda Ben Hadid
}

\section{- To cite this version:}

Ridha Touihri, Anas El Gallaf, Daniel Henry, Hamda Ben Hadid. Instabilities in a cylindrical cavity heated from below with a free surface. II. Effect of a horizontal magnetic field.. Physical Review E: Statistical, Nonlinear, and Soft Matter Physics, 2011, 84, pp.056303. 10.1103/PhysRevE.84.056303 . hal-00691094

\section{HAL Id: hal-00691094 \\ https://hal.science/hal-00691094}

Submitted on 11 Apr 2016

HAL is a multi-disciplinary open access archive for the deposit and dissemination of scientific research documents, whether they are published or not. The documents may come from teaching and research institutions in France or abroad, or from public or private research centers.
L'archive ouverte pluridisciplinaire HAL, est destinée au dépôt et à la diffusion de documents scientifiques de niveau recherche, publiés ou non, émanant des établissements d'enseignement et de recherche français ou étrangers, des laboratoires publics ou privés. 


\title{
Instabilities in a cylindrical cavity heated from below with a free surface. II. Effect of a horizontal magnetic field
}

\author{
R. Touihri, ${ }^{1}$ A. El Gallaf, ${ }^{2}$ D. Henry, ${ }^{2}$ and H. Ben Hadid ${ }^{2}$ \\ ${ }^{1}$ LAMSIN, ENIT, Campus Universitaire, Le Belvédère, BP 37, TN-1002 Tunis, Tunisia \\ ${ }^{2}$ Laboratoire de Mécanique des Fluides et d'Acoustique, CNRS/Université de Lyon, Ecole Centrale de Lyon/Université Lyon 1/INSA de Lyon, \\ ECL, 36 Avenue Guy de Collongue, FR-69134 Ecully Cedex, France
}

(Received 23 December 2010; published 8 November 2011)

\begin{abstract}
The effect of a constant and uniform horizontal magnetic field on the flow in a cylindrical cavity heated from below, with a free surface at the top, is numerically investigated. The azimuthal modes, which usually trigger convection in a cylinder, are changed by the horizontal magnetic field to oriented modes, either parallel or perpendicular to the magnetic field direction. The corresponding primary thresholds increase with the Hartmann number Ha. This increase, however, depends on the structure of the modes and is the weakest for the parallel modes and the strongest for the perpendicular modes. The changes that affect the evolution of the primary thresholds with the aspect ratio for nonzero Ha are also emphasized. The nonlinear evolution of the convection with a horizontal magnetic field is presented through bifurcation diagrams for different values of the Prandtl number Pr. For $\operatorname{Pr}=1$ and small values of $\mathrm{Ha}$, the structuring effect of the horizontal magnetic field, which involves modifications of the flow structures and bifurcation points, is put into light. Results are finally shown for smaller Pr values corresponding to liquid metals.
\end{abstract}

DOI: 10.1103/PhysRevE.84.056303

PACS number(s): 47.20.Bp

\section{INTRODUCTION}

The effect of a magnetic field on convection has been investigated in situations belonging to various domains, such as astrophysics, material processing, or, more recently, tokamak development. In crystal growth processes, the steady magnetic field is often used to control the convective flow in the melt. The desired effect of the magnetic field is to damp the convection and suppress the instabilities and, by the way, to improve the quality of the obtained crystals.

Ben Hadid et al. [1] and Ben Hadid and Henry [2,3] studied the effect of a constant magnetic field on the flow in laterally heated parallelepipedic or cylindrical cavities, corresponding to horizontal Bridgman crystal growth configurations. They considered different magnetic field orientations, as well as different situations for the parallelepipedic geometry: confined cavity, cavity with a free surface at the top, and cavity with the upper boundary subjected to surface tension. For the long cavities (length/height $=4$ ), the magnetic damping is found to be more effective for the vertical magnetic field. In addition, the effect of the magnetic field strongly depends on the configurations studied, i.e., on the cross-section shape and the boundary conditions. Alboussière et al. [4] analytically studied the influence of the cross-section shape on the magnetic damping in the case of long horizontal cavities. They have shown that, with electrically insulated walls, the damped convective velocity varies as a function of the Hartmann number as $\mathrm{Ha}^{-2}$ when the cross section has a horizontal plane of symmetry and as $\mathrm{Ha}^{-1}$ for nonsymmetrical shapes, whereas for conductive walls, the damped velocity always varies as $\mathrm{Ha}^{-2}$. More recently, Henry et al. [5] numerically studied the directional effect of a magnetic field on the onset of time-periodic convection in a laterally heated parallelepipedic cavity. They found that the critical Grashof number and the frequency at the Hopf bifurcation point exponentially increase with the Hartmann number, and that the vertical magnetic field is still the most efficient.
In the Rayleigh-Bénard situation, representative of vertical Bridgman growth configurations, different studies on the action of a steady magnetic field have been performed. Chandrasekhar [6] first made a linear analysis to study the effect of a vertical magnetic field on convective instabilities in a fluid layer confined by horizontal walls and heated from below, showing that the primary threshold varies as $\mathrm{Ha}^{2}$ and the wave number decreases as $\mathrm{Ha}^{-1 / 3}$. Benzid et al. [7] recently extended the study by considering the combined effect of a high frequency vibration and a constant magnetic field. They found that the Rayleigh-Bénard system submitted to gravity and vibrations is systematically stabilized by a magnetic field perpendicular to the horizontal walls. This stabilization by the magnetic field is, in particular, effective in microgravity conditions $(\mathrm{Ra}=0)$ where only vibrational $g$-jitter accelerations are involved. Touihri et al. [8] numerically investigated the effect of a constant and uniform magnetic field on the convection in a confined cylindrical cavity heated from below. They have shown that the vertical magnetic field is more efficient than the horizontal field, but that the horizontal field gives interesting modifications of the flow patterns and bifurcation diagrams due to the breaking of some symmetries. Houchens et al. [9] extended these results toward larger Hartmann numbers in the case of a vertical magnetic field. They developed two linear analyses: first, a hybrid approach, which combines an analytical solution for the Hartmann layer with a numerical solution for the rest of the liquid domain, and then a second approach, which involves an analytical asymptotic solution for large Ha. They concluded that the combination of the hybrid approach, the asymptotic analysis, and the fully numerical study of Touihri et al. [8] provides accurate linear results for values of Ha from zero to infinity. Walker et al. [10] also presented a linear analysis on the effect of a steady, uniform, vertical magnetic field on the buoyant convection during the liquid-encapsulated Czochralski growth process. Their calculations concerned two fluids: indium-phosphide 
(InP) melt and gallium-arsenide (GaAs) melt. They concluded that, for the weak magnetic fields, the critical Rayleigh number variation in both cases is close to that for the Rayleigh-Bénard instability in a cylindrical cavity. For the stronger fields, in contrast, the critical Rayleigh number for GaAs is higher than for InP because convective heat transfer in the GaAs base flow reduces the vertical temperature gradient.

In this paper, we present a numerical study on the effect of a constant and uniform horizontal magnetic field on the convection in a cylindrical cavity heated from below with a free surface at the top. The pure thermal situation $(\mathrm{Ha}=0)$ was treated by a linear approach by Vrentas et al. [11] and Dauby et al. [12] and extended in the companion paper [13] toward larger parameter ranges and nonlinear analyses. Our results on the effect of the horizontal magnetic field are first presented through stability diagrams giving the evolution of the primary thresholds as a function of the Hartmann number and the aspect ratio of the cavity. The nonlinear evolution of the convection is then given through bifurcation diagrams: the structuring effect of a weak magnetic field is studied for $\operatorname{Pr}=1$ and the further influence of moderate fields is considered for a small Pr value.

\section{PHYSICAL MODEL AND NUMERICAL METHODS}

We consider an incompressible liquid layer contained in a vertical cylindrical cavity that is placed in a constant magnetic field. The cavity has aspect ratio $A=R_{d} / H$, where $R_{d}$ is the radius of the cavity and $H$ is its height (Fig. 1). The lower end of the cylinder is assumed isothermal and held at the temperature $T_{b}$; a Newton law $-\lambda \partial_{z} T=h\left(T-T_{g}\right)$ expresses the heat exchange between the liquid free surface and the ambient gas ( $\lambda$ is the liquid thermal conductivity, $h$ the heat exchange coefficient, and $T_{g}$ the temperature of the ambient gas); and the vertical sidewalls are considered as adiabatic. The fluid is assumed to be Newtonian with constant physical properties (kinematic viscosity $v$, thermal diffusivity $\kappa$, electric conductivity $\sigma_{e}$, density $\rho$ ), except for the density in

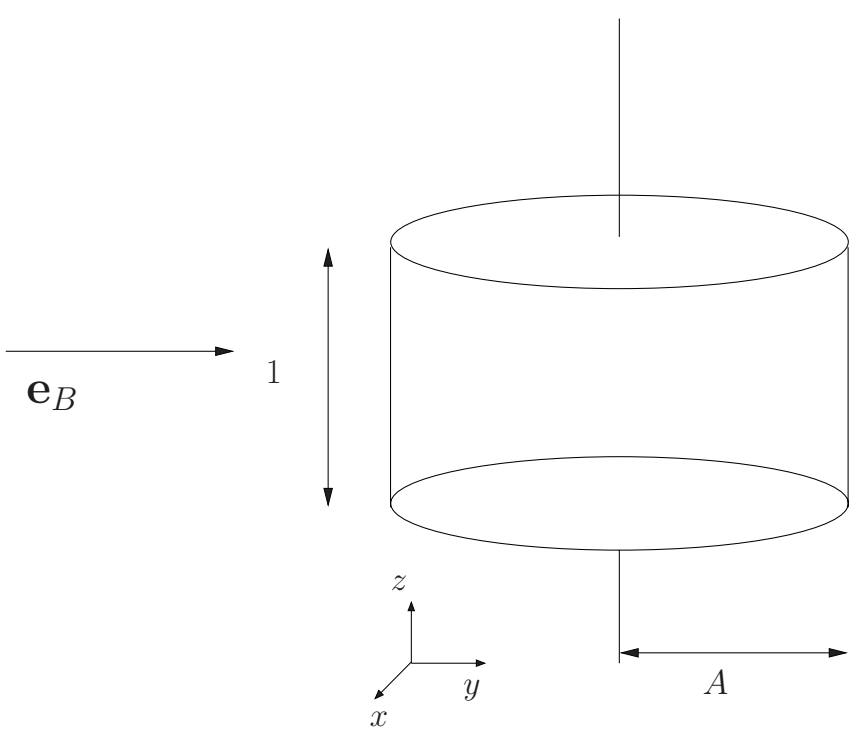

FIG. 1. Schematic diagram of the cylindrical cavity. the buoyancy term, which, in the Boussinesq approximation, depends linearly on temperature $\rho=\rho_{\text {ref }}\left[1-\beta\left(T-T_{\text {ref }}\right)\right]$, where $\beta$ is the thermal expansion coefficient, $T_{\text {ref }}$ is a reference temperature, and $\rho_{\text {ref }}$ is the value of the density at $T_{\text {ref. }}$. The free surface is flat and subject to a surface tension $\sigma$, which is assumed to vary linearly with the temperature $\sigma=\sigma_{\text {ref }}\left[1+\gamma\left(T-T_{\text {ref }}\right)\right]$, where $\sigma_{\text {ref }}$ is the surface tension at $T_{\text {ref }}$ and $\gamma$ is constant. According to Moreau [14], in most laboratory experiments using molten metals, the induced magnetic field is negligible, so that the applied magnetic field $\boldsymbol{B}=|B| \boldsymbol{e}_{B}$ can be considered as the effective magnetic field. The imposed thermal conditions generate a conductive rest state, which corresponds to a linear temperature profile along the vertical coordinate

$$
T(\bar{z})=-\frac{\mathrm{Bi}}{1+\mathrm{Bi}}\left(T_{b}-T_{g}\right) \frac{\bar{z}}{H}+T_{b},
$$

with a temperature at the upper surface of the layer $T_{t}=$ $-\mathrm{Bi}\left(T_{b}-T_{g}\right) /(1+\mathrm{Bi})+T_{b}$ and, thus, a temperature difference across the layer $\Delta T=T_{b}-T_{t}=\mathrm{Bi}\left(T_{b}-T_{g}\right) /(1+\mathrm{Bi})$. In these expressions, $\mathrm{Bi}$ is the Biot number defined as $\mathrm{Bi}=h H / \lambda$.

The convective motion is governed by the Navier-Stokes equations coupled to an energy equation. Using $H, H^{2} / \kappa$, $\kappa / H, \rho_{\text {ref }} \kappa^{2} / H^{2}, \Delta T, \kappa|B|$, and $\sigma_{e} \kappa|B| / H$ as scales for length, time, velocity, pressure, temperature, induced electric potential, and induced current, respectively, these equations take the following form:

$$
\begin{aligned}
& \nabla \cdot \boldsymbol{u}=0, \\
& \partial_{t} \mathbf{u}+(\mathbf{u} \cdot \nabla) \mathbf{u}=-\nabla p+\operatorname{Pr} \nabla^{2} \mathbf{u}+\operatorname{Pr} \operatorname{Ra} \theta \mathbf{e}_{z} \\
& +\operatorname{PrHa}^{2} \mathbf{j} \times \mathbf{e}_{B}, \\
& \partial_{t} \theta+(\mathbf{u} \cdot \nabla) \theta=\nabla^{2} \theta \text {, }
\end{aligned}
$$

where $\mathbf{e}_{z}$ and $\mathbf{e}_{B}$ are unit vectors in the vertical direction and in the direction of $\boldsymbol{B}$, respectively. In the equation of motion (2b), the body force $\operatorname{PrHa}^{2} \boldsymbol{j} \times \boldsymbol{e}_{B}$ is the Lorentz force that results from the interaction between the induced electric current density $\boldsymbol{j}$ and the applied magnetic field $\boldsymbol{B}$. The dimensionless electric current density $\boldsymbol{j}$ is given by Ohm's law for a moving fluid:

$$
\boldsymbol{j}=-\nabla \phi_{e}+\boldsymbol{u} \times \boldsymbol{e}_{B},
$$

where $\phi_{e}$ is the dimensionless electric potential. By combining the continuity equation for $\boldsymbol{j}, \boldsymbol{\nabla} \cdot \boldsymbol{j}=0$, and Ohm's law [Eq. (3)], we obtain the dimensionless equation governing the electric potential:

$$
\nabla^{2} \phi_{e}=\boldsymbol{e}_{B} \cdot(\nabla \times \boldsymbol{u})
$$

We impose no-slip boundary conditions along the rigid bottom and lateral walls. The lateral walls are thermally insulating, while a constant temperature is maintained on the bottom. Along the rigid bottom at $z=0$, we thus obtain $u=v=w=$ $0, \theta=1$, and along the lateral walls at $r=A, u=v=w=$ $\partial_{r} \theta=0$. Along the free surface, the normal velocity $w$ is zero and the stress equilibrium gives the boundary conditions for 
$u$ and $v$, whereas the heat exchange law gives the boundary condition for $\theta$. At the free surface at $z=1$, we thus obtain

$$
\frac{\partial u}{\partial z}-\operatorname{Ma} \frac{\partial \theta}{\partial x}=\frac{\partial v}{\partial z}-\operatorname{Ma} \frac{\partial \theta}{\partial y}=\frac{\partial \theta}{\partial z}+\operatorname{Bi} \theta+1=w=0 .
$$

The nondimensional parameters arising from the scaling of the equations are the Rayleigh number $\operatorname{Ra}=\left(\beta g \Delta T H^{3} / \nu \kappa\right)$, the Prandtl number $\operatorname{Pr}=v / \kappa$, the Marangoni number $\mathrm{Ma}=$ $\left(\Delta T H \sigma_{\text {ref }} \gamma / \rho \nu \kappa\right)$, the Biot number already defined, and the Hartmann number $\mathrm{Ha}=|B| H \sqrt{\sigma_{e} / v \rho_{m}}$. The dimensionless variables are the velocity vector $\boldsymbol{u}=(u, v, w)$ [defined along the $(x, y, z)$ coordinates], the pressure $p$, and the temperature $\theta$, and the dimensionless conductive temperature profile is given by $\theta(z)=1-z$.

The governing equations are solved in the threedimensional cylindrical domain using a spectral element method. The solutions are obtained by time integration or by steady state solving through a Newton method. A continuation method allows us to follow the solution branches as well as the primary or secondary bifurcation points. Details on the methods are given in the companion paper [13].

Compared to the case without magnetic field (see the companion paper [13]), the calculation of the perturbation kinetic energy budget at critical parameter values will now involve the energy dissipation related to the magnetic forces $E_{\text {magn }}$, which is defined as

$$
E_{\text {magn }}=\operatorname{Re}\left(\int_{\Omega} \operatorname{PrHa}^{2}\left(\boldsymbol{j}^{\prime} \times \boldsymbol{e}_{B}\right) \boldsymbol{u}^{\prime *} d \Omega\right) .
$$

The perturbation kinetic energy at threshold, normalized by $-E_{\mathrm{visc}}=\left|E_{\mathrm{visc}}\right|$, will be

$$
E_{\text {shear }}^{\prime}+E_{\text {buoy }}^{\prime}+E_{\text {Mar }}^{\prime}+E_{\text {magn }}^{\prime}=1 .
$$

For primary thresholds and for $\mathrm{Ma}=0$, we get

$$
E_{\text {buoy }}^{\prime}+E_{\text {magn }}^{\prime}=1 \text {. }
$$

If $\mathrm{Ra}$ is factored out of the buoyancy energy term so that $E_{\text {buoy }}^{\prime}=\operatorname{Ra} E_{\text {buoy }}^{\prime \prime}$, Eq. (8) at marginal stability can be rewritten as

$$
\operatorname{Ra}_{c} E_{\text {buoy }}^{\prime \prime}=1-E_{\text {magn }}^{\prime},
$$

which leads to

$$
\frac{\mathrm{Ra}_{c}}{\operatorname{Ra}_{c, 0}}=\overbrace{\left(\frac{E_{\text {buoy }_{0}}^{\prime \prime}}{E_{\text {buoy }}^{\prime \prime}}\right)}^{R_{\text {buoy }}} \overbrace{\left(1-E_{\text {magn }}^{\prime}\right)}^{R_{\text {magn }}},
$$

where the values with the subscript 0 refer to the case where $\mathrm{Ha}=0$.

\section{RESULTS}

The results presented in this paper have been obtained for particular values of the Biot and Marangoni numbers, $\mathrm{Bi}=100$ and $\mathrm{Ma}=0$, corresponding to an almost fixed temperature at the upper surface and to the absence of surface tension. We consider a constant horizontal magnetic field along the $y$ direction, which has both a stabilizing effect on the
TABLE I. Tests of numerical accuracy for $\operatorname{Pr}=0.0286$ : The secondary bifurcation threshold $\mathrm{Ra}_{S_{2}}$ and Hopf bifurcation threshold $\mathrm{Ra}_{H_{1}}$ of Fig. 14 are given as a function of the mesh size.

\begin{tabular}{lccc}
\hline \hline & \multicolumn{3}{c}{$\operatorname{Mesh}\left(N_{x y} \times N_{z}\right)$} \\
\cline { 2 - 4 } & $(337 \times 11)$ & $(521 \times 13)$ & $(745 \times 17)$ \\
\hline $\mathrm{Ra}_{S_{2}}$ & 1739 & 1750 & 1747 \\
$\mathrm{Ra}_{H_{1}}$ & 1646 & 1632 & 1632 \\
\hline \hline
\end{tabular}

convection and an influence on the flow structures due to symmetry changes.

We will first define the different primary modes that are obtained when a horizontal magnetic field is applied, and then study the variation of their thresholds with respect to the aspect ratio $A$ and the Hartmann number Ha. Concerning the nonlinear dynamics, we will compute bifurcation diagrams for $\mathrm{Pr}=1$ and $\mathrm{Ha}=1$ and analyze how the horizontal magnetic field modifies the characteristics of the bifurcations. Finally, we will consider smaller values of the Prandtl number (as $\operatorname{Pr}=0.0286$ ) characteristic of molten metal, and present the dynamics in this case, without and with an applied horizontal magnetic field.

The same mesh refinement, corresponding to $\left(N_{x y} \times N_{z}\right)=$ $(337 \times 11)$, has been used for all the calculations in this paper. This mesh has been validated in the companion paper [13] for $\operatorname{Pr}=1$ and without magnetic field. Precision tests have been performed for $\operatorname{Pr}=0.0286$ on the values of the secondary $S_{2}$ and Hopf $H_{1}$ bifurcation points (Table I). We see that the mesh corresponding to $(337 \times 11)$ points gives a precision of about $1 \%$ for these thresholds. Concerning the influence of the magnetic field, the range of Ha values used in this paper is limited, so that we can keep the same mesh refinement. Indeed, for the primary thresholds, which did not need such a mesh refinement for $\mathrm{Ha}=0$, the Hartmann number does not exceed the value $\mathrm{Ha}=30$, and for the nonlinear calculations, the values used are still smaller and correspond to $\mathrm{Ha}=1$ and 10 .

\section{A. Onset of convection: Evolution of primary thresholds and flow structures}

As mentioned in the companion paper [13], the way the convection will develop above the critical threshold will depend on the symmetries of the system. The horizontal magnetic field defines a preferential direction and then does not allow the azimuthal invariance found for the convection in a cylinder. More precisely, when a horizontal magnetic field is applied, the initial $O(2)$ symmetry group, valid for convection in a cylindrical cavity with a free surface, is changed to a $Z_{2} \times Z_{2} \equiv D_{2}$ group. This $D_{2}$ group corresponds to the reflection symmetries with respect to two vertical planes containing the vertical axis of the cylinder, the plane containing the direction of the magnetic field denoted as $P_{\|}$, and the plane perpendicular to this direction denoted as $P_{\perp}$.

Due to these symmetry changes, the Fourier modes found without magnetic field are changed when a horizontal magnetic field is applied. For example, as illustrated in Fig. 2, the first three Fourier modes $m=0,1$, and 2 give five distinct modes denoted as $m=0 / 2_{\|}, 2^{a}, 0 / 2_{\perp}, 1_{\|}$, and $1_{\perp}$. The $m=1$ mode, which was defined to within a rotation without magnetic 


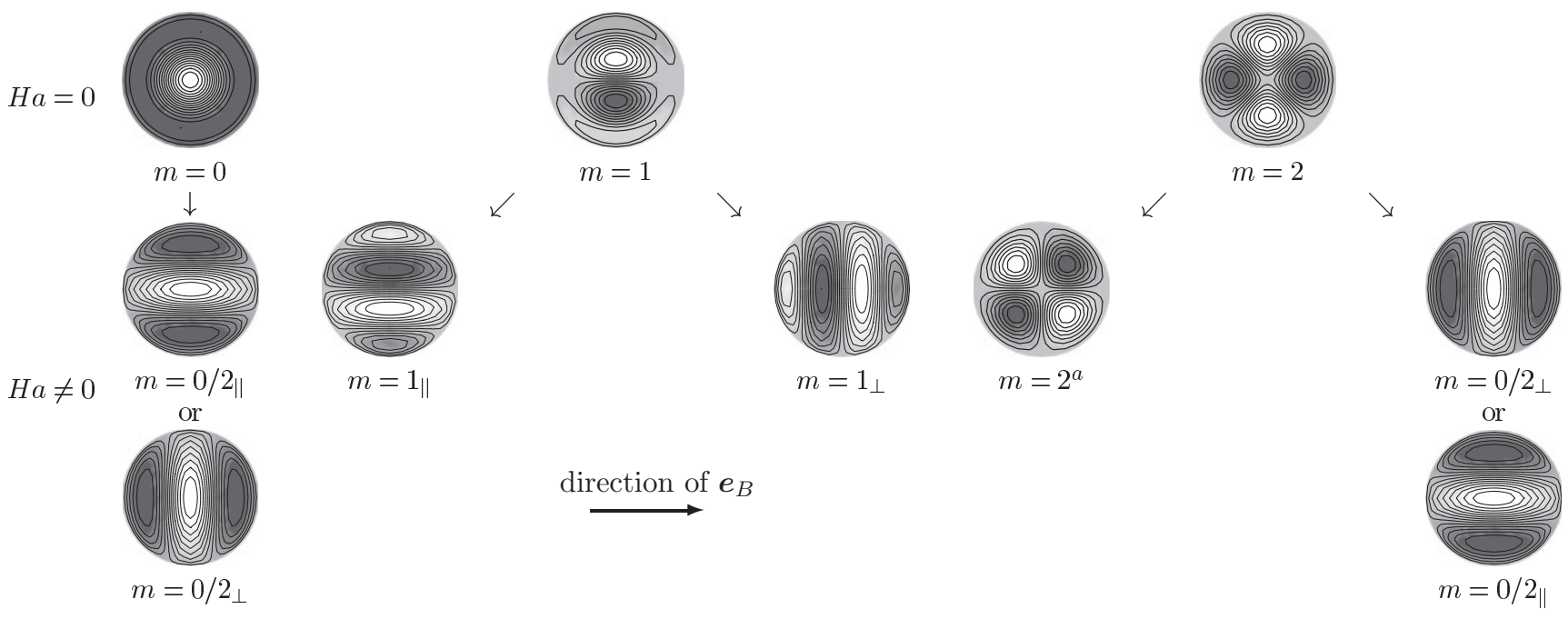

FIG. 2. Variation of the first three Fourier modes when a horizontal magnetic field is applied. The figure shows the three modes $m=0,1$, and 2 for $\mathrm{Ha}=0$ (the modes $m=1$ and 2 are defined to within a rotation), and the different possible modes $m=0 / 2_{\|}, 2^{a}, 0 / 2_{\perp}, 1_{\|}$, and $1_{\perp}$ for $\mathrm{Ha} \neq 0$. Both modes $m=0$ and 2 can evolve into $m=0 / 2_{\|}$or $0 / 2_{\perp}$ modes; it is in fact the more unstable of these two modes without magnetic field that will evolve into the $m=0 / 2_{\|}$mode, and the other mode will evolve into the $m=0 / 2_{\perp}$ mode. The plots give the isovalues of the vertical velocity at midheight in the cavity.

field, gives the two modes $m=1_{\|}$and $1_{\perp}$, which have rolls with their axis, respectively, parallel and perpendicular to the magnetic field direction. These new modes break one of the reflection symmetries of the $D_{2}$ group and keep the other symmetry. Concerning the two other modes $m=0$ and 2, there is a connection between their evolution when a horizontal magnetic field is applied. Indeed, the axisymmetric mode $m=0$ (which can not keep the axisymmetry with a horizontal magnetic field) is changed into either an $m=0 / 2_{\|}$mode or an $m=0 / 2_{\perp}$ mode, whereas the $m=2$ mode (defined to within a rotation without magnetic field) gives two modes: a first mode denoted as $m=2^{a}$, which has its two zero vertical velocity planes oriented along the main horizontal directions $x$ and $y$ (this mode is the only one that breaks both symmetries with respect to the $P_{\|}$and $P_{\perp}$ planes), and a second mode, which is either the $m=0 / 2_{\perp}$ mode or the $m=0 / 2_{\|}$mode. These changes of the modes $m=0$ and 2 due to the horizontal magnetic field are depicted in Fig. 3, which shows the variation with the aspect ratio of the critical thresholds for the modes $m=0$ and 2 (without magnetic field) and for the modes $m=0 / 2_{\|}, 0 / 2_{\perp}$, and $2^{a}$ when the magnetic field corresponds to $\mathrm{Ha}=3$. These thresholds $\mathrm{Ra}_{c}$ are expressed as $\mathrm{Ra}_{c}-\mathrm{Ra}_{0}$, i.e., the difference with respect to the threshold $\mathrm{Ra}_{0}$ of the $m=0$ mode for $\mathrm{Ha}=0$. We see that the critical curves of the $m=0$ and 2 modes intersect at $A \approx 0.9$, whereas those of the $m=0 / 2_{\|}, 0 / 2_{\perp}$, and $2^{a}$ modes do not intersect. The reason is that, without magnetic field, the two modes $m=0$ and 2 have different symmetries, which allows their critical curves to intersect, whereas the modes $m=0 / 2_{\|}$and $0 / 2_{\perp}$ (which are issued from either the $m=0$ mode or the $m=2$ mode) have identical symmetries as they both keep the symmetries of the $D_{2}$ group, and their critical curves can not intersect. Figure 3 also clearly shows that the $m=0 / 2_{\|}$mode comes from the $m=2$ mode for $A<0.9$ and from the $m=0$ mode for $A>0.9$. This indicates that this $m=0 / 2_{\|}$mode, which is little stabilized because the magnetic field is parallel to the roll axis, comes from the one of the two $m=0$ and 2 modes, which is first critical for $\mathrm{Ha}=0$. The $m=0 / 2_{\perp}$ mode, in contrast, comes from the one of the two modes that is the more stable for $\mathrm{Ha}=0$, i.e., the $m=0$ mode for $A<0.9$ and the $m=2$ mode for $A>0.9$.

The influence of the cylinder aspect ratio on the primary thresholds in a situation with a horizontal magnetic field is shown in Fig. 4 for $\mathrm{Ha}=3$. We see that the dominant modes are those corresponding to rolls with their axis parallel to the magnetic field direction, namely, the $m=1_{\|}$mode for

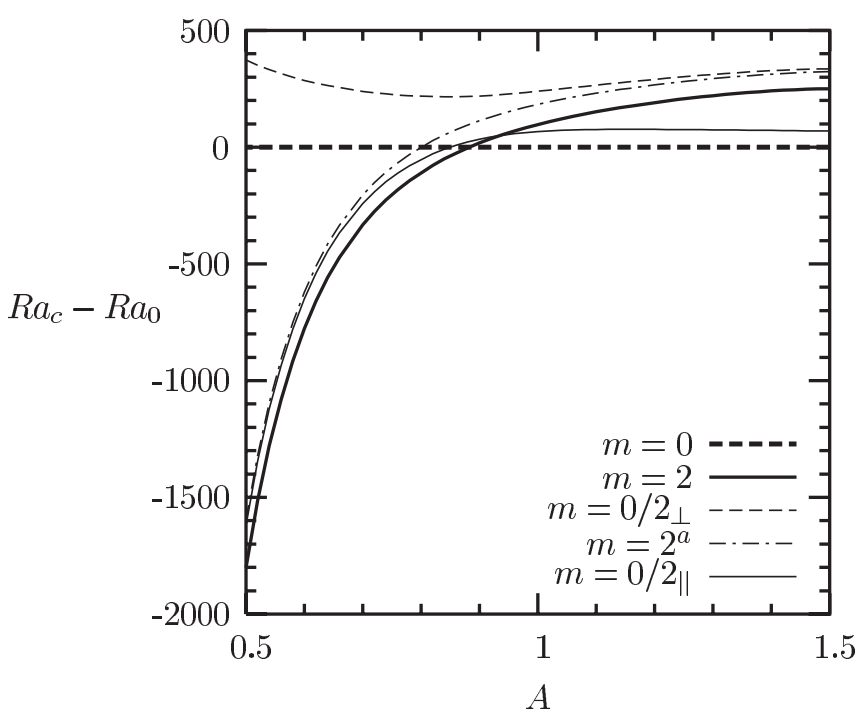

FIG. 3. Primary thresholds for the modes $m=0$ and 2 without magnetic field $(\mathrm{Ha}=0)$ and for the modes $m=0 / 2_{\|}, 0 / 2_{\perp}$, and $2^{a}$ into which they evolve when a horizontal magnetic field is applied (Ha $=3$ for this figure). These thresholds $\mathrm{Ra}_{c}$ are given as a function of the cavity aspect ratio $A$ and expressed by $\mathrm{Ra}_{c}-\mathrm{Ra}_{0}$, i.e., the difference with the threshold $\mathrm{Ra}_{0}$ of the $m=0$ mode for $\mathrm{Ha}=0$. 


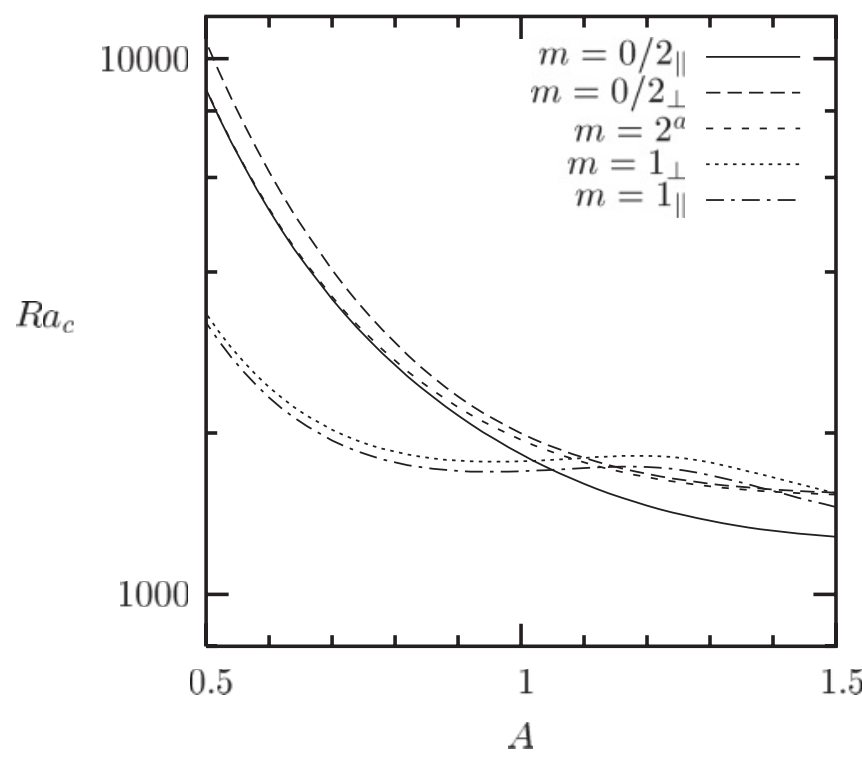

FIG. 4. Variation of the thresholds $\mathrm{Ra}_{c}$ for the primary modes as a function of the aspect ratio $A$ in a case with a horizontal magnetic field $(\mathrm{Ha}=3)$.

$0.5<A<1.05$ and the $m=0 / 2_{\|}$mode for $1.05<A<1.5$. In contrast, the modes corresponding to rolls with their axis perpendicular to the magnetic field direction have larger thresholds. At $\mathrm{Ha}=3$, the critical curves for the modes $m=1_{\|}$ and $1_{\perp}$ have quite similar variations with the aspect ratio $A$. Minimum values are reached at $A \approx 0.9$ and a change of curvature (corresponding to a change in the number of rolls from 1 to 3 ) takes place at $A \approx 1.075$. As seen before, the critical curves for the three $m=0 / 2_{\|}, 2^{a}$, and $0 / 2_{\perp}$ modes do not intersect. The curve for the $m=2^{a}$ mode (which has been generated from the curve of the $m=2$ mode at $\mathrm{Ha}=0$ ) is in-between the curves for the $m=0 / 2_{\|}$and $0 / 2_{\perp}$ modes. It is very close to the curve of the $m=0 / 2_{\|}$mode for $A<0.8$ and very close to that of the $m=0 / 2_{\perp}$ mode for $A>1.2$, whereas it evolves differently in the intermediate range of $A$, i.e., around the intersection point of the $m=0$ and 2 modes at $\mathrm{Ha}=0$. Note that these curves obtained for $\mathrm{Ha}=3$ are very close to each other. They will indeed become more and more separate for higher values of the Hartmann number.

The influence of the Hartmann number Ha on the critical thresholds $\mathrm{Ra}_{c}$ for the primary modes is shown more in details in Fig. 5 for $A=1.5$. All the different curves emerging from the first three thresholds at $\mathrm{Ha}=0$ (successively for the $m=0,1$, and 2 modes) increase with $\mathrm{Ha}$, which indicates a general stabilizing influence of the magnetic field. The modes, however, are differently stabilized. The modes corresponding to rolls with their axis perpendicular to the magnetic field direction are strongly stabilized; those corresponding to rolls with their axis parallel to the magnetic field direction have a weaker stabilization, and the $m=2^{a}$ mode has an intermediate stabilization. Note that the more selective stabilization by the horizontal magnetic field is obtained for the $m=1$ mode, with the largest initial stabilization for the $m=1_{\perp}$ mode and the weakest for the $m=1_{\|}$mode. The stronger stabilization of the $m=0 / 2_{\|}$mode compared to the $m=1_{\|}$mode explains the shift of the intersection point of the dominant modes

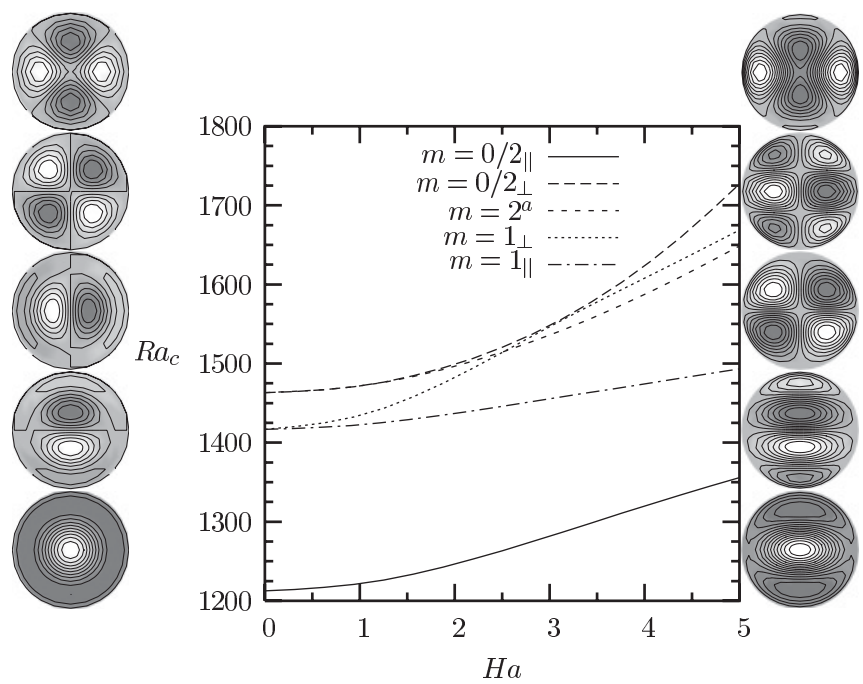

FIG. 5. Stabilization of the first three critical modes by a horizontal magnetic field in a cavity with $A=1.5$. The thresholds $\mathrm{Ra}_{c}$ are given as a function of $\mathrm{Ha}$ for $\mathrm{Ha} \leqslant 5$. Insets show vertical velocity contours at midheight in the cavity for the different modes in order of appearance at $\mathrm{Ha}=1$ (left insets) and $\mathrm{Ha}=5$ (right insets).

toward stronger values of $A$ as $\mathrm{Ha}$ is increased: for example, this intersection point is changed from $A=0.9$ for $\mathrm{Ha}=0$ to $A=1.05$ for $\mathrm{Ha}=3$. These different evolutions of the modes with increasing Ha may induce crossings between the critical curves, except that the curves of $m=0 / 2_{\|}, 2^{a}$, and $0 / 2_{\perp}$ modes can not cross each other. Note that Fig. 5 also clearly shows how the modes are changed when the horizontal magnetic field is applied for $A=1.5$. The dominant $m=0$ mode is changed into an $m=0 / 2_{\|}$mode, the $m=1$ mode, defined to within a rotation, gives both the $m=1_{\|}$and $1_{\perp}$ modes, the thresholds of which progressively separate when Ha is increased, and the $m=2$ mode, also defined to within a rotation, gives the $m=2^{a}$ and $0 / 2_{\perp}$ modes, which more slowly separate. From the plots given as insets for $\mathrm{Ha}=1$ and 5 in Fig. 5, we see that the flow structures for $\mathrm{Ha}=1$ have specific orientations (either parallel or perpendicular to the magnetic field direction), but are not much changed compared to the case without magnetic field (the mode $m=0$ is almost axisymmetric, and the modes that have split are still similar). In contrast, for $\mathrm{Ha}=5$, we see that all the modes have evolved. The parallel modes have rolls (with their axis parallel to $B$ ) that are elongated in the direction of $B$, two rolls for the $m=0 / 2_{\|}$mode and three rolls for the $m=1_{\|}$ mode. The $m=1_{\perp}$ mode has rolls with a common axis (the $x$ axis, which is perpendicular to $B$ ), a main roll with a size equal to the diameter of the cavity, and two, less expected, counter-rotating rolls closer to the lateral wall. This flow structure looks quite similar to that of an $m=3$ mode. Finally, the modes coming from the $m=2$ modes have less changed, although the two-roll structure of the $m=0 / 2_{\perp}$ mode becomes more visible.

The influence of the Hartmann number on the primary thresholds is also shown in Fig. 6 for $A=1$. In that case, the first destabilized mode at $\mathrm{Ha}=0$ is the $m=1$ mode, before the $m=0$ and 2 modes. The stabilization of the modes can be observed in this figure in a larger range of Ha. The parallel 


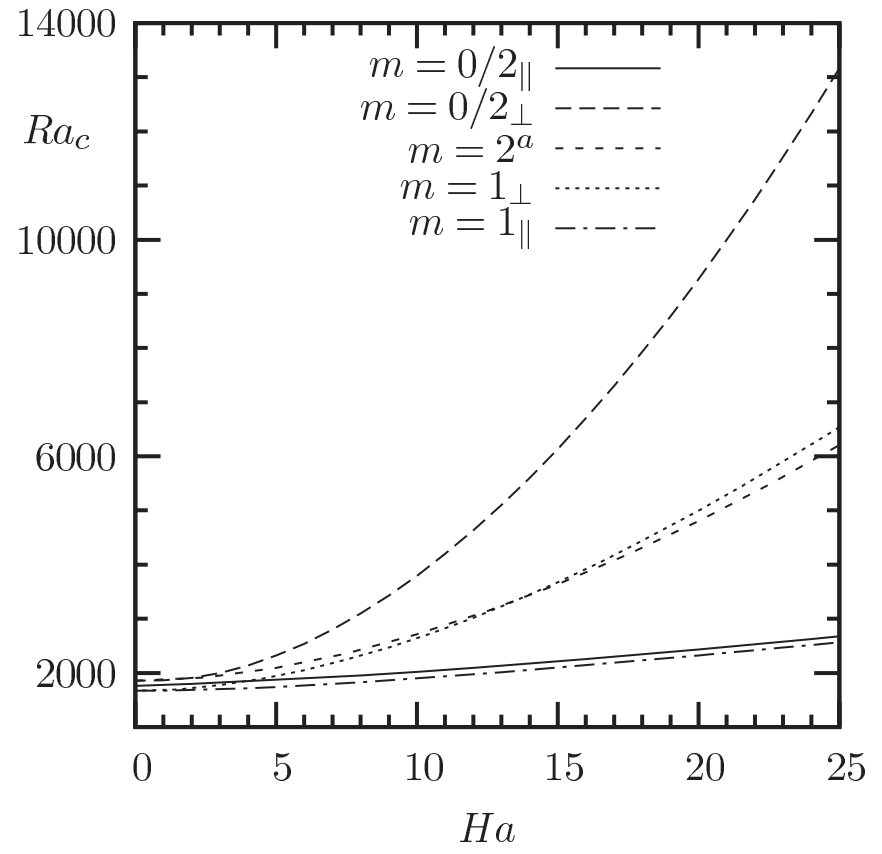

FIG. 6. Stabilization of the first three critical modes by a horizontal magnetic field in a cavity with $A=1$. The thresholds $\mathrm{Ra}_{c}$ are given as a function of $\mathrm{Ha}$ for $\mathrm{Ha} \leqslant 25$.

modes $m=1_{\|}$and $0 / 2_{\|}$are similarly and weakly stabilized, with almost parallel variations of the critical curves. The $m=2^{a}$ and $1_{\perp}$ modes are better stabilized and their critical curves also have close evolutions. Finally, the best stabilization is obtained for the $m=0 / 2_{\perp}$ mode. These different critical curves can be fitted by expressions of the form

$$
\frac{\mathrm{Ra}_{c}-\mathrm{Ra}_{c, 0}}{\mathrm{Ra}_{c, 0}} \approx a \times 10^{-3} \mathrm{Ha}^{b} .
$$

Such polynomial expressions were found to fit the numerical data quite accurately over the range of Ha studied. The values of the prefactor $a$ and the exponent $b$ are given in Table II for the different instability modes. We can see that the powers of Ha are between 1.35 and 1.95. The smallest powers of Ha are obtained for the parallel modes; in fact, the $m=1_{\|}$mode has a larger value of the exponent $b$ than the $m=0 / 2_{\|}$mode, but this effect is counterbalanced by a smaller value of the prefactor $a$. Larger powers of $\mathrm{Ha}$ are obtained for the intermediate modes, and here also the larger value of $b$ for the $m=2^{a}$ mode compared to the $m=1_{\perp}$ mode is counterbalanced by a smaller value of $a$. Finally, the strongest power of $\mathrm{Ha}$ is obtained for the $m=0 / 2_{\perp}$ mode.

TABLE II. Values of the prefactor $a$ and the power of $\mathrm{Ha} b$ for the polynomial expressions of the form $\left[\mathrm{Ra}_{c}-\mathrm{Ra}_{c}(\mathrm{Ha}=0)\right] / \mathrm{Ra}_{c}(\mathrm{Ha}=$ $0) \approx a \times 10^{-3} \mathrm{Ha}^{b}$, which best fit the stability curves as a function of Ha for the different instability modes. The results have been obtained for $A=1, \mathrm{Bi}=100$, and $\mathrm{Ma}=0$.

\begin{tabular}{cccccc}
\hline \hline & $m=1_{\|}$ & $m=0 / 2_{\|}$ & $m=2^{a}$ & $m=1_{\perp}$ & $m=0 / 2_{\perp}$ \\
\hline$a$ & 4.95 & 6.74 & 8.35 & 12.12 & 11.45 \\
$b$ & 1.45 & 1.35 & 1.75 & 1.70 & 1.95 \\
\hline \hline
\end{tabular}

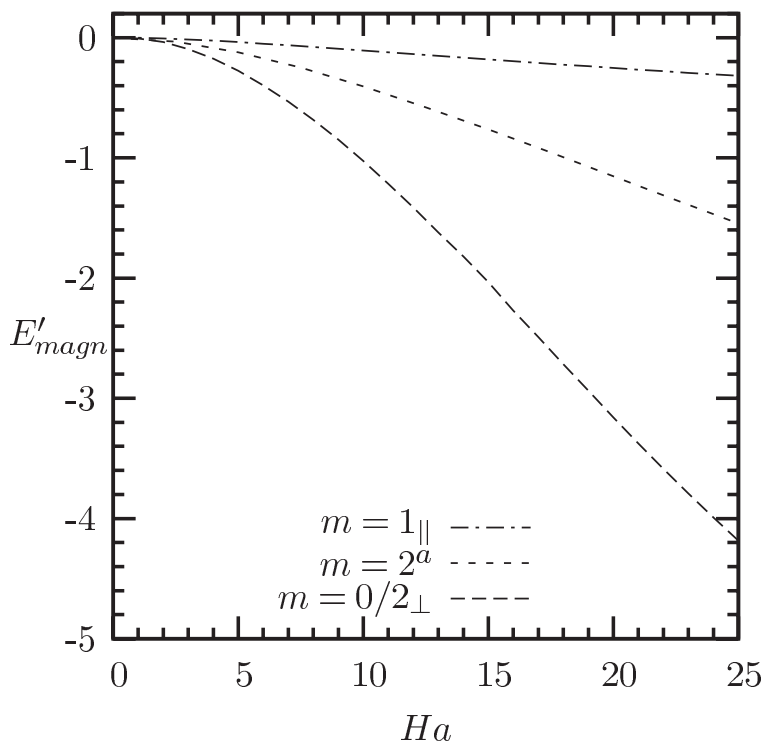

FIG. 7. Variation of the magnetic energy contribution $E_{\text {magn }}^{\prime}$ to the perturbation kinetic energy budget as a function of the Hartmann number for the modes $m=1_{\|}, 2^{a}$, and $0 / 2_{\perp}$ in a cavity with $A=1$.

We will analyze these three different evolutions of the thresholds by the calculation of the perturbation kinetic energy budget for the modes $m=1_{\|}, 2^{a}$, and $0 / 2_{\perp}$. In Fig. 7, we give the variation of the magnetic energy contribution $E_{\text {magn }}^{\prime}$ with the Hartmann number for these three modes. As expected, $E_{\text {magn }}^{\prime}$ is negative, indicating a stabilizing influence of the magnetic energy term, and this influence increases as $\mathrm{Ha}$ is increased. $\mathrm{Up}$ to $\mathrm{Ha}=25$, this magnetic energy term remains small for the case of the $m=1_{\|}$mode, reaching about $30 \%$ of the viscous energy term, but it is much stronger for the two other cases, reaching about $150 \%$ of the viscous energy term for the case of the $m=2^{a}$ mode and even more than $400 \%$ for the case of the $m=0 / 2_{\perp}$ mode. The energetic contributions to the critical Rayleigh number, $R_{\text {buoy }}$ and $R_{\text {magn }}$, calculated according to Eq. (10) are given as a function of Ha for the same three modes in Fig. 8. In all cases, the main energetic contribution to the critical Rayleigh number is the magnetic contribution, but the buoyancy contribution also plays a role. The magnetic contribution increases with $\mathrm{Ha}$, but at $\mathrm{Ha}=25$, the slope of the variation has already begun to decrease. The values reached at $\mathrm{Ha}=25$ are close above $1.3,2.5$, and 5 for the $m=1_{\|}, 2^{a}$, and $0 / 2_{\perp}$ modes, respectively. The buoyancy contribution, which is induced by the changes of the flow structure with the magnetic field, is weaker. Its initial variation is slow and can even be slightly decreasing (down to 0.98) for the $m=$ $0 / 2_{\perp}$ modes. The values reached at $\mathrm{Ha}=25$ are, respectively, $1.16,1.31$, and 1.37 for the three modes, which give relative contributions $R_{\text {buoy }} / R_{\text {magn }}$ equal to $88.3 \%, 51.5 \%$, and $26.4 \%$. Thus, the relative contribution of the buoyancy energy is the strongest in the cases where the stabilization is the weakest.

The symmetry changes from $O(2)$ to $D_{2}$, which occur when a horizontal magnetic field is applied, will affect the characteristics of the primary bifurcations. The transcritical bifurcation associated with the axisymmetric $m=0$ mode will remain transcritical as the two new possible modes $m=0 / 2_{\|}$and $0 / 2_{\perp}$ do not break any symmetry of the $D_{2}$ 


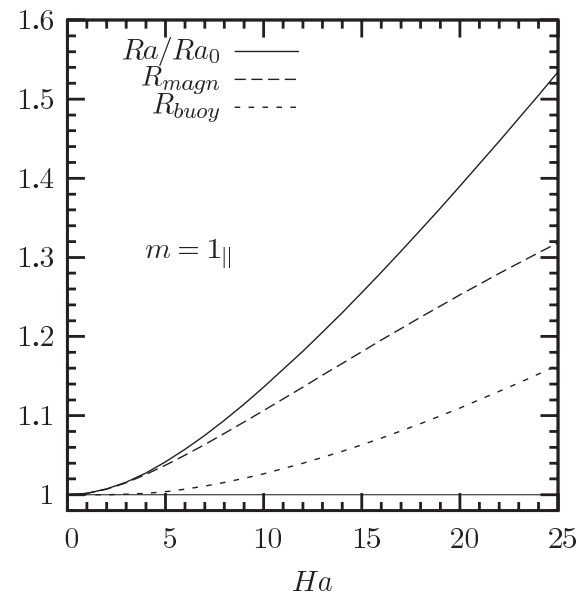

(a)

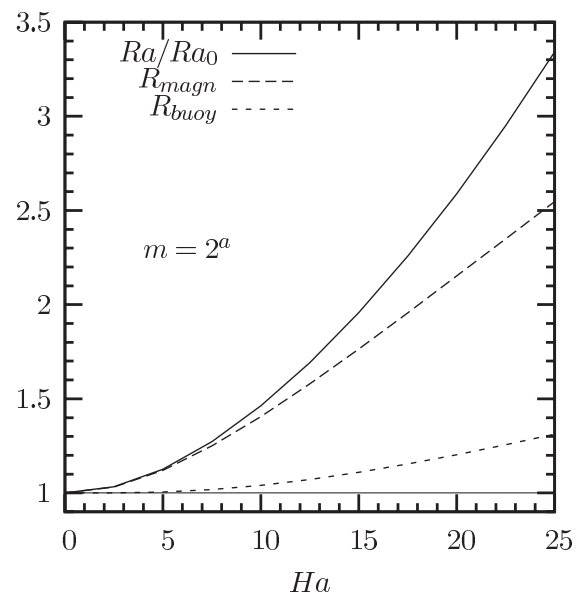

(b)

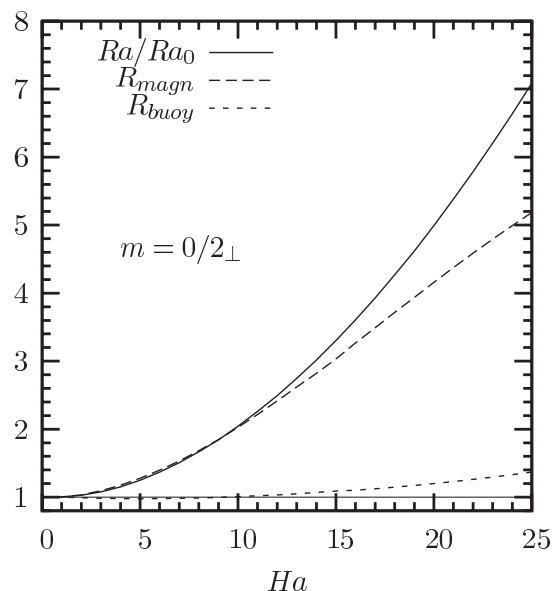

(c)

FIG. 8. Variation of the energetic contributions to the critical Rayleigh number $R_{\text {buoy }}$ and $R_{\text {magn }}$ [calculated according to (10)] as a function of Ha for the modes (a) $m=1_{\|}$, (b) $m=2^{a}$, and (c) $m=0 / 2_{\perp}$ in a cavity with $A=1$.

group. The same type of modes are triggered at one of the bifurcations issued from the $m=2$ threshold at $\mathrm{Ha}=0$, so that this bifurcation is also transcritical. These transcritical bifurcations, associated with a single eigenvalue, generate two different branches: a stable supercritical branch and an unstable subcritical branch, which will be later stabilized at a saddle-node point. In contrast, the bifurcations associated with the $m=1_{\|}, 1_{\perp}$, and $2^{a}$ modes are pitchfork bifurcations as they break at least one of the $D_{2}$ symmetries. These bifurcations are no more circular pitchfork bifurcations (as they were without magnetic field) because of the loss of the rotational invariance, and they are associated with a single eigenvalue. These symmetry changes will also affect the secondary bifurcations, in particular, those which, at $\mathrm{Ha}=0$, connect the axisymmetric solution to the $m=0 / 2$ solutions. As the axisymmetric solution is already transformed into an $m=0 / 2$ solution by the horizontal magnetic field, these secondary bifurcations will disappear and be replaced by imperfect bifurcations. More precisions will be given in the next section during the study of the bifurcation diagrams.

\section{B. Dynamics of the convection for a weak magnetic field at $\operatorname{Pr}=1$}

In the companion paper [13], the nonlinear dynamics of the convection in a cylindrical cavity was presented in a case where the primary mode was axisymmetric. Two axisymmetric solution branches with different dynamics were obtained. In this paper, we first consider the same configuration corresponding to $A=1.5, \operatorname{Pr}=1, \mathrm{Ma}=0$, and $\mathrm{Bi}=100$, and impose a constant horizontal magnetic field in the $y$ direction. A small value of the Hartmann number, $\mathrm{Ha}=1$, is chosen so as to focus on the modifications induced by the symmetry changes from $O(2)$ to $D_{2}$.

The vertical velocity at the center of the cavity $w_{0}$ was plotted in the bifurcation diagrams without magnetic field because all the solutions defined to within a rotation (those initiated by the $m=1$ and 2 modes) appeared as a single point in this diagram. $w_{0}$ is less adapted in the case with magnetic field because the different solutions that separate under the influence of the magnetic field remain close in this representation, particularly for small Hartmann numbers. In order to best portray the features of the bifurcation diagram with magnetic field, we then chose to plot the vertical velocity $w_{l}$ at a fixed spatial location outside the cylinder axis. Finally, note that we use solid (dashed) lines in the bifurcation diagrams to indicate linearly stable (unstable) steady solutions.

\section{Bifurcation diagram}

The thermally stratified motionless basic flow is stable up to $\mathrm{Ra}_{P_{\|}}=1222$, where a transcritical bifurcation to $m=0 / 2_{\|}$ states occurs. These states are characterized by two rolls, the axes of which are parallel to the magnetic field direction. At this point, two different branches of solutions are produced. The branch with $w_{l}<0\left(w_{l}>0\right)$ is supercritical ( subcritical) and corresponds to states with downflow (upflow) along the cylinder axis. As in the case without magnetic field, the subcritical branch quickly undergoes a saddle-node bifurcation where it is stabilized. The corresponding hysteresis is too small to be visible on the bifurcation diagrams. The next primary bifurcations that affect the basic flow are first those related to the $m=1_{\|}$and $1_{\perp}$ modes, which result from the splitting of the bifurcation to the $m=1$ mode obtained at $\mathrm{Ha}=0$. These bifurcations occur at $\mathrm{Ra}_{P_{1}}=1422$ and $\mathrm{Ra}_{P_{1_{\perp}}}=1434$, respectively. The splitting of the $m=2$ mode obtained at $\mathrm{Ha}=0$ then gives two other primary bifurcations related to the modes $m=2^{a}$ and $0 / 2_{\perp}$, which occur at $\operatorname{Ra}_{P_{2} a}=1471$ and $\operatorname{Ra}_{P_{2_{\perp}}}=1472$, respectively.

As expected from the linear results, $\mathrm{Ra}_{P_{1_{\|}}}<\mathrm{Ra}_{P_{1_{\perp}}}$. Concerning the other bifurcations, we have to note that, in the case chosen $(A=1.5)$, the threshold corresponding to the $m=0$ mode is below that corresponding to the $m=2$ mode for $\mathrm{Ha}=0$. It is why the $m=0 / 2_{\|}$mode comes from the $m=0$ mode, whereas the $m=2^{a}$ mode (less stabilized) and the $m=0 / 2_{\perp}$ mode are obtained from the $m=2$ mode. Note also that because of the small value of $\mathrm{Ha}(\mathrm{Ha}=1)$, the modes that split still have close thresholds. All the branches obtained 


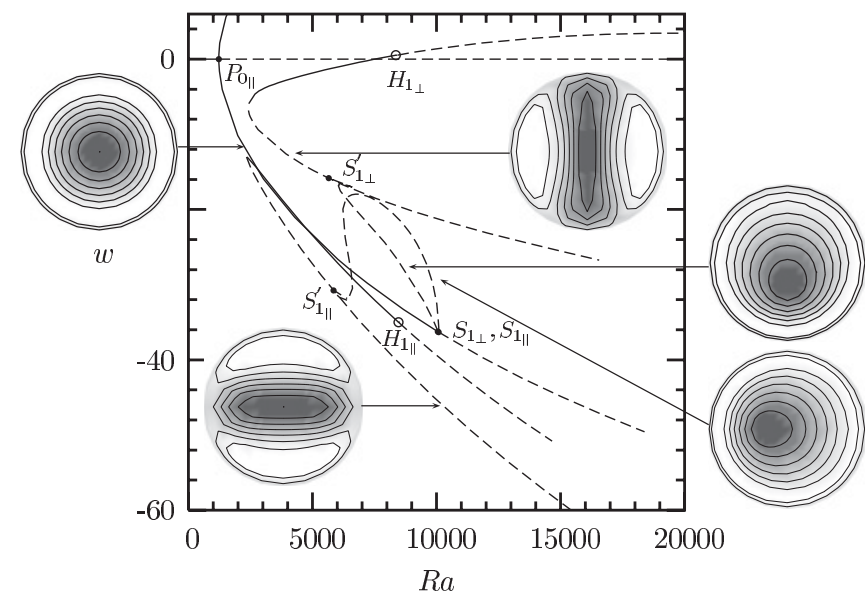

FIG. 9. Bifurcation diagram for the supercritical part of the axisymmetric branch for a cavity with $A=1.5$ when a weak magnetic field $(\mathrm{Ha}=1)$ is applied $(\mathrm{Pr}=1)$. This diagram involves the splitting of an original pitchfork secondary bifurcation. Insets show vertical velocity contours at midheight in the cavity.

from the primary modes $m=1_{\|}, 1_{\perp}, 2^{a}$, and $0 / 2_{\perp}$ have been found to remain unstable in the Ra range studied.

The supercritical $m=0 / 2_{\|}$branch shown in Fig. 9 remains stable up to $\mathrm{Ra}_{S_{1}}=10061$ where a secondary pitchfork bifurcation associated with $m=1_{\perp}$ perturbations occurs. Another pitchfork bifurcation now associated with $m=1_{\|}$ perturbations occurs for a slightly larger Rayleigh number at $\mathrm{Ra}_{S_{1 \|}}=10087$ [see Fig. 10(a)]. In fact, by changing the symmetry of the problem from $O(2)$ to $D_{2}$, the horizontal magnetic field induces the splitting of the double multiplicity zero eigenvalue at $\mathrm{Ra}_{S_{1}}$, which was associated with an $m=1$ mode defined to within a rotation. The circular pitchfork bifurcation at $\mathrm{Ra}_{S_{1}}$ is thus split into two successive pitchfork bifurcations at $\mathrm{Ra}_{S_{1_{\perp}}}$ and $\mathrm{Ra}_{S_{1_{\|}}}$. This splitting is shown in Fig. 10(b), which has been obtained by following $S_{1_{\perp}}$ and $S_{1_{1}}$ down to $\mathrm{Ha}=0$. We clearly see how the horizontal magnetic field progressively splits the thresholds of the $m=1_{\perp}$ and $1_{\|}$

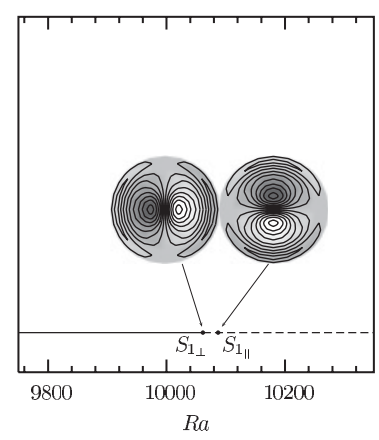

(a)

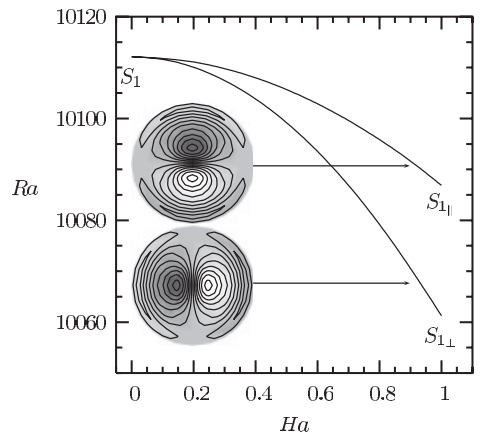

(b)
FIG. 10. (a) Zoom of the two pitchfork secondary bifurcation points $S_{1_{\perp}}$ and $S_{1_{\|}}$on the supercritical branch of the $m=0 / 2_{\|}$solution for $\mathrm{Ha}=1$ together with the corresponding critical perturbations. (b) Splitting of the pitchfork secondary bifurcation point $\mathrm{Ra}_{S_{1}}$ into the two points $\mathrm{Ra}_{S_{1_{\perp}}}$ and $\mathrm{Ra}_{S_{1_{\|}}}$when a horizontal magnetic field is applied $(0 \leqslant \mathrm{Ha} \leqslant 1)$. Branches of $m=0 / 1_{\perp}$ and $0 / 1_{\|}$solutions will be generated at these points, respectively. The results have been obtained for $A=1.5$ and $\operatorname{Pr}=1$.

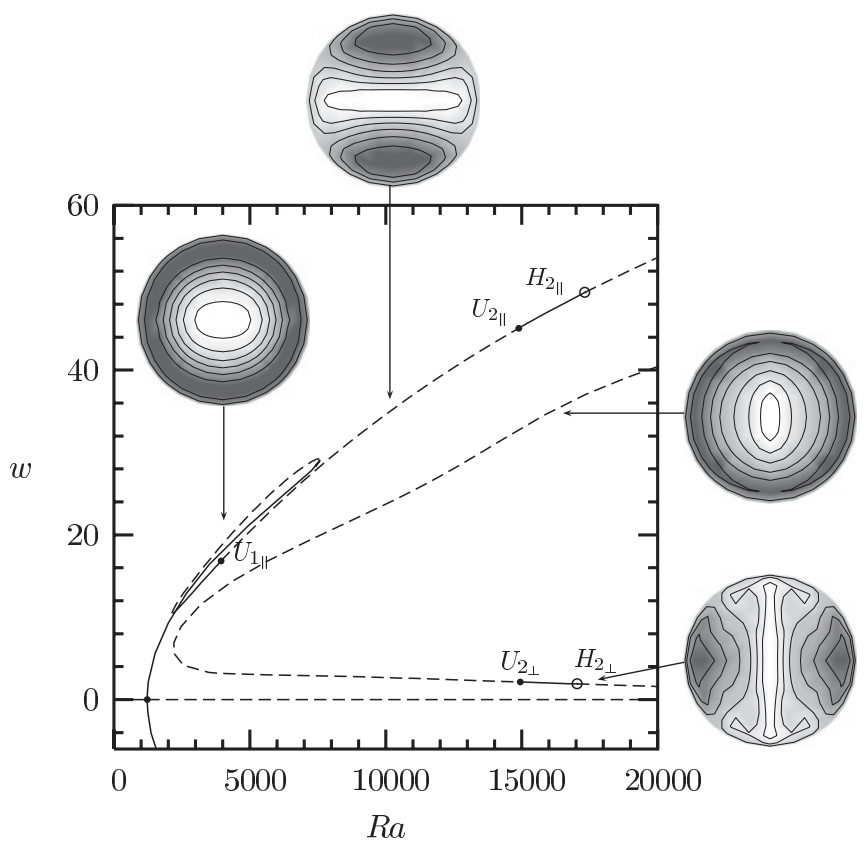

FIG. 11. Bifurcation diagram for the subcritical part of the axisymmetric branch for a cavity with $A=1.5$ when a weak magnetic field $(\mathrm{Ha}=1)$ is applied $(\mathrm{Pr}=1)$. The complex behavior is the result of the unfolding of an original subcritical circular pitchfork secondary bifurcation. Insets show vertical velocity contours at midheight in the cavity.

modes as Ha is increased. Note that both thresholds decrease as Ha is increased and that the decrease is smaller for the $m=1_{\|}$ mode. As was the bifurcation at $S_{1}$, these bifurcations at $S_{1_{\perp}}$ and $S_{1_{\|}}$are subcritical. They produce unstable branches of $m=$ $0 / 1$ states, which differ by their symmetry properties as they break a different symmetry of the $D_{2}$ group. These branches evolve in a similar way as the $m=0 / 1$ branch in the case without magnetic field and eventually connect with $m=0 / 2$ solution branches at $\mathrm{Ra}_{S_{1_{\perp}^{\prime}}^{\prime}}=5657$ and $\mathrm{Ra}_{S_{1_{\|}^{\prime}}^{\prime}}=5848$. During this evolution, the $m=0 / 1$ solutions progressively recover both symmetries of the $D_{2}$ group. The new $m=0 / 2_{\perp}$ and $0 / 2_{\|}$ branches acquire stability for decreasing $\mathrm{Ra}$ at saddle-node bifurcations at $\mathrm{Ra}=2428$ (almost identical values for the two branches), and remain stable up to Hopf bifurcation points at $\mathrm{Ra}_{H_{1_{\perp}}}=8362$ and $\mathrm{Ra}_{H_{1_{\|}}}=8462$, respectively.

Except for the very small interval between $P_{0_{\|}}$and the accompanying saddle-node bifurcation, the subcritical $m=$ $0 / 2_{\|}$branch, shown in Fig. 11, remains stable up to $\mathrm{Ra}=7573$, where the curve reverses direction at a saddle-node point. Note that this initial stability range is much reduced compared to the case without magnetic field, where the loss of stability occurred at $\mathrm{Ra}_{S_{2}}=12513$. The branch is further stabilized at a second saddle-node point at $\mathrm{Ra}=2123$, and becomes unstable again at $\mathrm{Ra}_{U_{1 \|}}=3942$ before a restabilization at $\mathrm{Ra}_{U_{2}}=14881$. The branch is then definitively destabilized via a Hopf bifurcation at $\mathrm{Ra}_{H_{2 \|}}=17322$. All the solutions on this branch are $m=0 / 2 \|$ states, but the nonaxisymmetry of these states is more and more revealed as we progress along the branch. Compared to the case without magnetic field, we can see that these states belong now to a primary branch and 


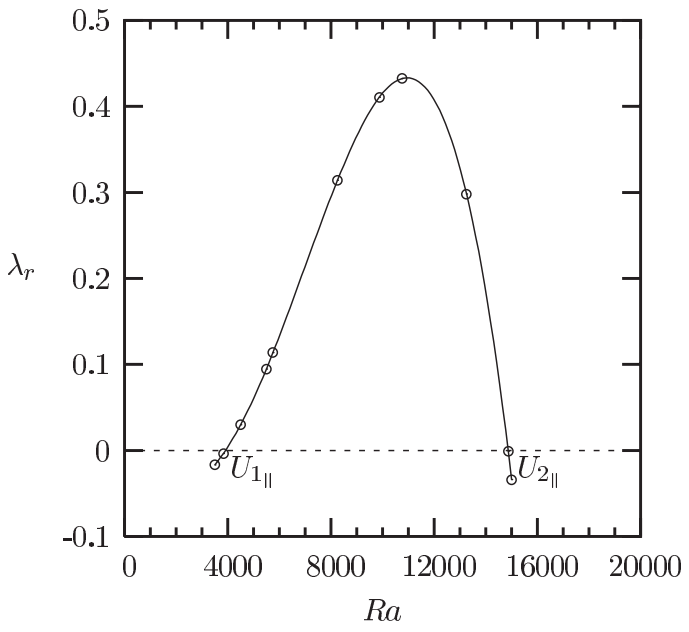

(a)

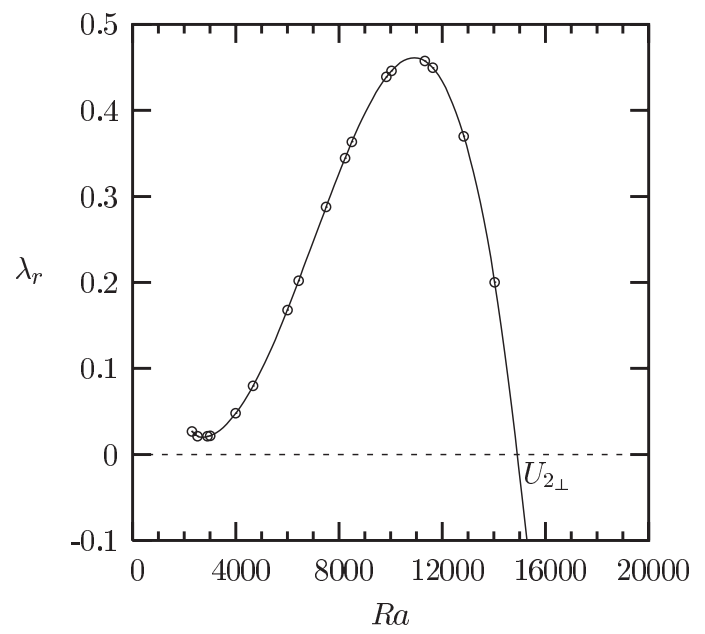

(b)

FIG. 12. Growth rate of the critical perturbations that stabilize the (a) $m=0 / 2_{\|}$and (b) $m=0 / 2_{\perp}$ branches at $U_{2_{\|}}$and $U_{2_{\perp}}$, respectively, in the bifurcation diagram of Fig. 11 . The parameters are $A=1.5, \mathrm{Ha}=1, \operatorname{Pr}=1$.

that this branch is characterized by the presence of saddle-node points. We will later see that these characteristics are typical of a subcritical circular pitchfork bifurcation, which becomes imperfect. In the present case, the imperfection is connected to the horizontal magnetic field, which, by breaking the rotational invariance, changes the symmetry from $O(2)$ to $D_{2}$. Note also that, as in the case without magnetic field, the destabilization and restabilization of the $m=0 / 2_{\|}$branch at $\mathrm{Ra}_{U_{1 \|}}$ and $\mathrm{Ra}_{U_{2} \|}$, respectively, is connected to the same perturbation. The amplification rate of this perturbation, shown in Fig. 12(a), has a similar bell shape as for $\mathrm{Ha}=0$. There exists also another branch, which is disconnected from the main branch and corresponds to $m=0 / 2_{\perp}$ states. This branch reverses direction at a saddle-node point corresponding to a small value of $\mathrm{Ra}$, but remains unstable and acquires stability only beyond a steady bifurcation at $\mathrm{Ra}_{U_{2}}=14942$ before a further destabilization via a Hopf bifurcation at $\mathrm{Ra}_{H_{2}}=17029$. The plot of the amplification rate of the critical perturbation involved at $\mathrm{Ra}_{U_{2}}$ is given in Fig. 12(b) for the branch portion containing $U_{2}{ }_{\perp}$ (the critical perturbation corresponds to an $m=2^{a}$ mode). The evolution of the amplification rate looks similar to what was obtained on the $m=0 / 2_{\|}$branch. The amplification rate, however, remains positive for the small values of Ra close to the saddle node, so that the $m=0 / 2_{\perp}$ branch is not stabilized in this Ra range.

We can summarize the results obtained for $\mathrm{Ma}=0, \mathrm{Bi}=$ $100, \operatorname{Pr}=1$ in a cylinder with aspect ratio $A=1.5$ submitted to a magnetic field with $\mathrm{Ha}=1$. Convection appears at a transcritical bifurcation as an $m=0 / 2_{\|}$mode, i.e., as a couple of rolls aligned with the magnetic field direction. The supercritical branch goes through two close bifurcation points at which two distinct branches of $m=0 / 1_{\|}$and $0 / 1_{\perp}$ states appear. These solutions evolve until $m=0 / 2_{\|}$and $0 / 2_{\perp}$ states are reached, which are further stabilized at saddle-node points. The subcritical branch remains stable up to a saddle-node point and regains stability at a second saddle-node point. It is accompanied by a disconnected branch of $m=0 / 2_{\perp}$ states that reverses direction at a saddle-node point close to the saddle-node point of the subcritical branch but remains unstable. We then see that the change in symmetry from $O(2)$ to $D_{2}$ due to the magnetic field induces the splitting of the circular pitchfork bifurcation at $\mathrm{Ra}_{S_{1}}$ (which connected the axisymmetric solution with $m=0 / 1$ states) into two successive pitchfork bifurcations (at which one of the $D_{2}$ symmetries is lost). In contrast, the circular pitchfork bifurcation at $\mathrm{Ra}_{S_{2}}$, which connected the axisymmetric solution with $m=0 / 2$ states, disappears because the primary flow with magnetic field is already an $m=0 / 2$ state. The corresponding evolution of the branches with the appearance of a saddle-node point and a disconnected branch will be studied in the next section. Note, finally, that the point at which the supercritical primary branch becomes unstable is only slightly modified by the magnetic field at $\mathrm{Ha}=1$ (the relative variation between $\mathrm{Ra}_{S_{1}}$ and $\mathrm{Ra}_{S_{1}}$ is less than $0.3 \%$ ), whereas for the subcritical branch, the corresponding relative variation between the bifurcation point at $\mathrm{Ra}_{S_{2}}$ and the saddle-node point appearing with magnetic field is about $40 \%$.

\section{Unfolding of the subcritical circular pitchfork bifurcations}

One of the more interesting changes that was observed in the nonlinear dynamics of the convection problem when a weak horizontal magnetic field is applied, i.e., when the symmetry of the problem is changed from $O(2)$ to $D_{2}$, is the unfolding of the subcritical circular pitchfork bifurcation where a secondary branch of $m=0 / 2$ states appears for $\mathrm{Ha}=0$. The amplitude equations that describe this unfolding are given by

$$
\frac{d A}{d t}=\mu A+|A|^{2} A-|A|^{4} A+\epsilon,
$$

where $A$ is a complex variable, $\mu$ is the threshold distance $\left(\mathrm{Ra}-\mathrm{Ra}_{c}\right) / \mathrm{Ra}_{c}$, and $\epsilon(0<\epsilon \ll 1)$ measures the weak influence of the horizontal magnetic field. With $A$ expressed as $A=R e^{i \phi}$ (with $R>0$ ), we get

$$
\begin{gathered}
\frac{d R}{d t}=\mu R+R^{3}-R^{5}+\epsilon \cos \phi, \\
\frac{d \phi}{d t}=-\frac{\epsilon \sin \phi}{R} .
\end{gathered}
$$



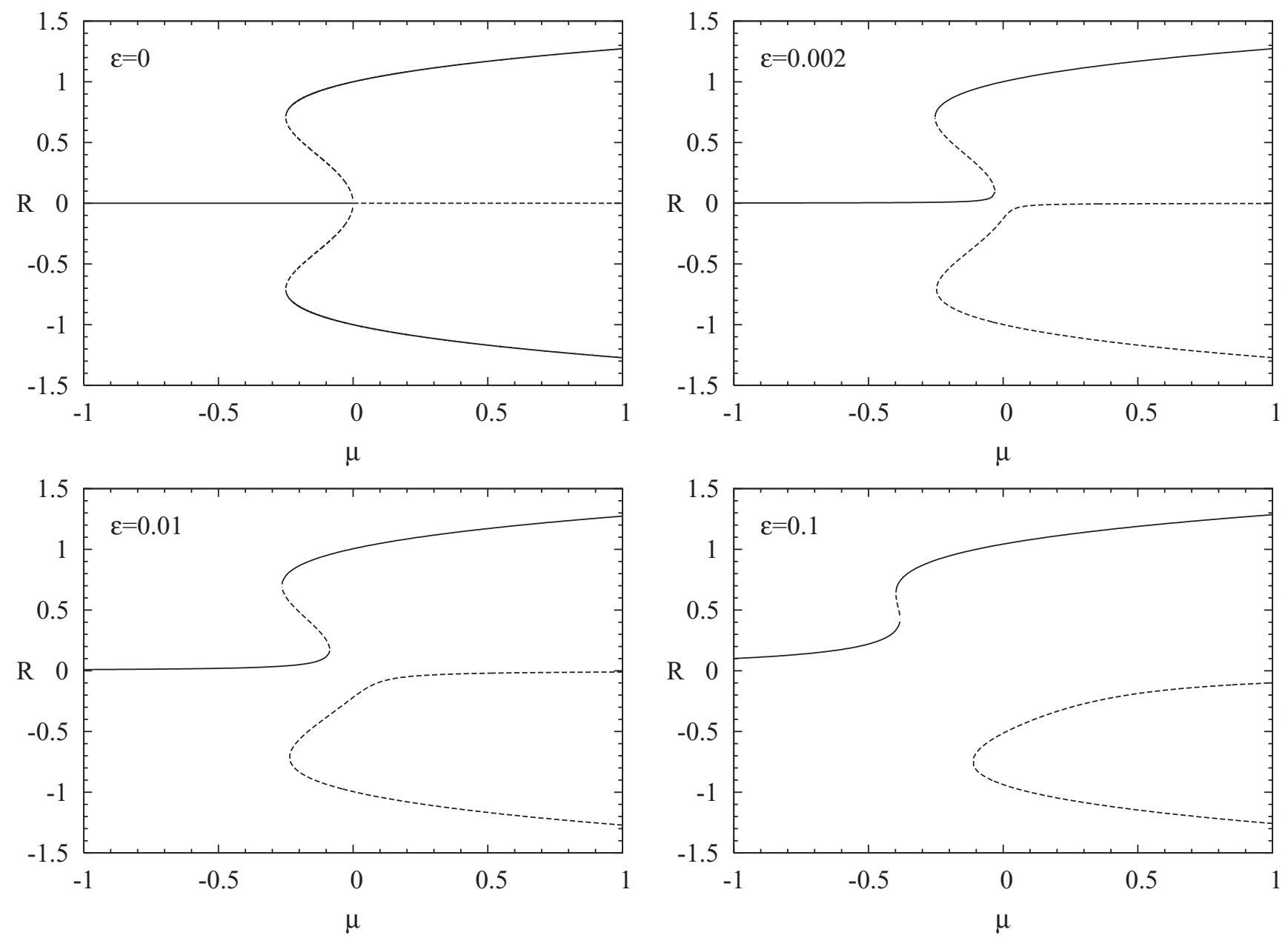

FIG. 13. Theoretical model showing the unfolding of a subcritical circular pitchfork bifurcation. The bifurcation diagrams give the amplitude $R$ as a function of the continuation parameter $\mu$ for different values of the imperfection parameter $\epsilon$. For $\epsilon=0$, the bifurcation is perfect and produces a circle of equivalent solutions. For $\epsilon \neq 0$, the bifurcation becomes imperfect and the changes in the diagram increase with increasing $\epsilon$.

According to (14), for $\epsilon=0$, the zeros of these equations are those of $\mu R+R^{3}-R^{5}=0$ and are defined for any $\phi \in[0,2 \pi]$. Moreover, the direction of $\phi$ is a neutral direction for these zeros. For $\epsilon \neq 0$, the zeros are only obtained for $\phi=0$ and $\pi$. For $\phi=0$, they are solutions of $\mu R+R^{3}-R^{5}+\epsilon=$ 0 and stable in the direction of $\phi$, whereas for $\phi=\pi$, they are solutions of $\mu R+R^{3}-R^{5}-\epsilon=0$ and unstable in the direction of $\phi$. All the zeros for $\phi=0$ and $\pi$ can also be considered as those of $\mu R+R^{3}-R^{5}+\epsilon=0$, if we choose $R \geqslant 0$ for $\phi=0$ and $R \leqslant 0$ for $\phi=\pi$. The solutions are represented in this way in Fig. 13.

The change of the subcritical circular pitchfork bifurcation when $\epsilon$ is increased is shown in Fig. 13. For $\epsilon=0$ (case without horizontal magnetic field), there exist circles of equivalent solutions defined to within a rotation $\phi$ [represented on the diagram by the two solutions for $\phi=0(R>0)$ and $\phi=\pi$ $(R<0)]$. We see that a circle of unstable solutions appears subcritically at a circular pitchfork bifurcation at $\mu=0$, and these solutions are stabilized at a saddle-node point. For $\epsilon \neq 0$ (case with a horizontal magnetic field), only two branches of solutions are kept, the branch for $\phi=0(R>0)$ and the branch for $\phi=\pi(R<0)$, and these branches are now disconnected due to the disappearance of the bifurcation at $\mu=0$. The branch with $R>0$, which is connected with the stable part of the basic solution at $\epsilon=0$, keeps the stability properties of the corresponding solutions at $\epsilon=0$ because this branch is attractive in the $\phi$ direction. For a small value of $\epsilon$ (as $\epsilon=0.002$ ), this branch has two saddle-node points; it is one time unstable beyond the first saddle-node point and retrieves stability beyond the second saddle-node point. In contrast, the other branch with $R<0$ (connected with the unstable part of the basic solution at $\epsilon=0$ ) is one time more unstable than the corresponding solutions at $\epsilon=0$ because it is now unstable in the $\phi$ direction. The former subcritical part of the branch becomes two time unstable in continuity with the part coming from the basic solution (at small negative $R$ ), and the part of the branch beyond the saddle-node point becomes one time unstable. When $\epsilon$ is further increased, the two branches become more and more separate due to the strong changes of the curves in the vicinity of $\mu=0$. The two saddle-node points on the upper branch also get closer and will collapse close above $\epsilon=0.1$, giving a continuously stable upper branch. At this stage, the initial characteristics of the system at $\epsilon=0$ are completely lost. 


\section{Dynamics of the convection in a liquid metal}

The influence of a horizontal magnetic field is now considered for the convection in a liquid metal characterized by a small Prandtl number $(0.01<\operatorname{Pr}<0.05)$. The particular value $\operatorname{Pr}=0.0286$ corresponding to liquid gallium has been chosen. Two cases will be presented in the following sections, a first case (for $A=1.5$ ) corresponding to a transcritical primary bifurcation and a second case (for $A=0.5$ ) corresponding to a supercritical primary bifurcation.

\section{Magnetic field effect on the convection induced at a transcritical primary bifurcation}

The bifurcation diagram obtained without magnetic field $(\mathrm{Ha}=0)$ for the convection in a cavity of aspect ratio $A=$ 1.5 for $\mathrm{Pr}=0.0286, \mathrm{Bi}=100$, and $\mathrm{Ma}=0$ is presented in Fig. 14. As shown in the companion paper [13], the primary bifurcation for $A=1.5$ is a transcritical bifurcation associated with a single eigenvalue, which occurs at $\operatorname{Ra}_{P_{0}}=1213$. Two distinct stable branches of axisymmetric solutions appear at this point: a subcritical branch with upflow at the center of the cavity and a supercritical branch with downflow at the center of the cavity. The supercritical $m=0$ branch is stable up to $\mathrm{Ra}_{S_{1}}=1391$, where a circular pitchfork bifurcation associated with a couple of $m=2$ perturbations occurs. The resulting circle of stable $m=0 / 2$ branches (defined to within a rotation) is further destabilized via a Hopf bifurcation at $\mathrm{Ra}_{H_{1}}=1646$. In contrast, the subcritical $m=0$ branch is destabilized by a couple of $m=3$ perturbations at $\operatorname{Ra}_{S_{2}}=1739$. The resulting circle of $m=0 / 3$ branches is stable in a small Ra range, up to $\operatorname{Ra}_{S_{2}^{\prime}}=1742$, where it is destabilized by a shifted $m=3$

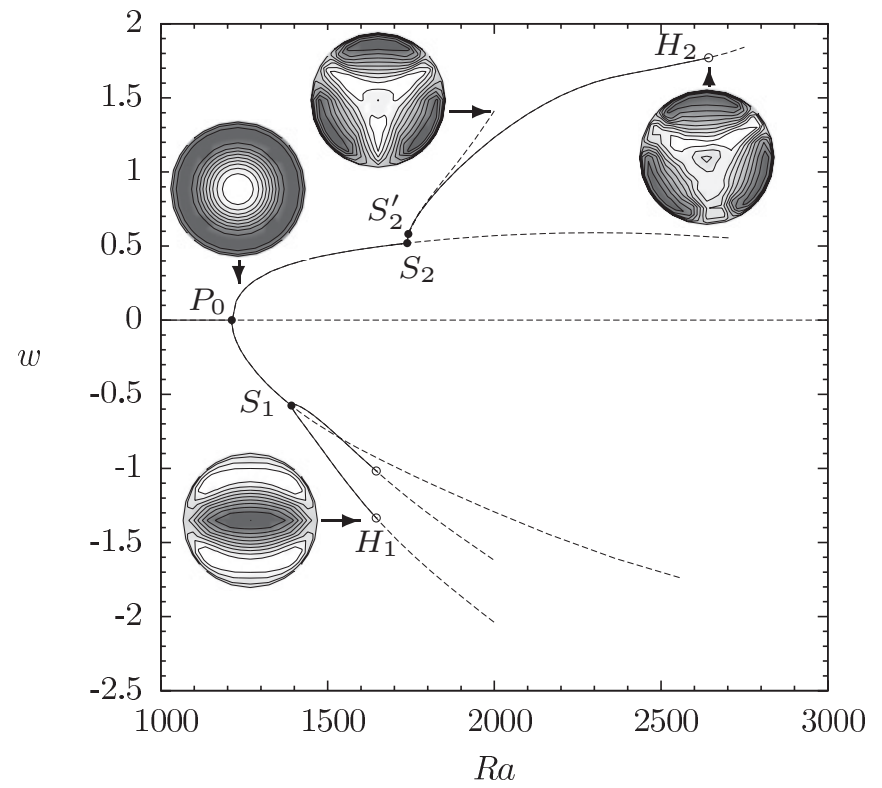

FIG. 14. Bifurcation diagram for a liquid metal $(\mathrm{Pr}=0.0286)$ in a cavity with $A=1.5$ in a case without magnetic field $(\mathrm{Ha}=0)$. On the supercritical axisymmetric branch, transition to an $m=0 / 2$ branch beyond a circular pitchfork bifurcation $S_{1}$. On the subcritical axisymmetric branch, transitions to $m=0 / 3$ states. Hopf bifurcations finally destabilize the steady flow branches. Insets show vertical velocity contours at midheight in the cavity.

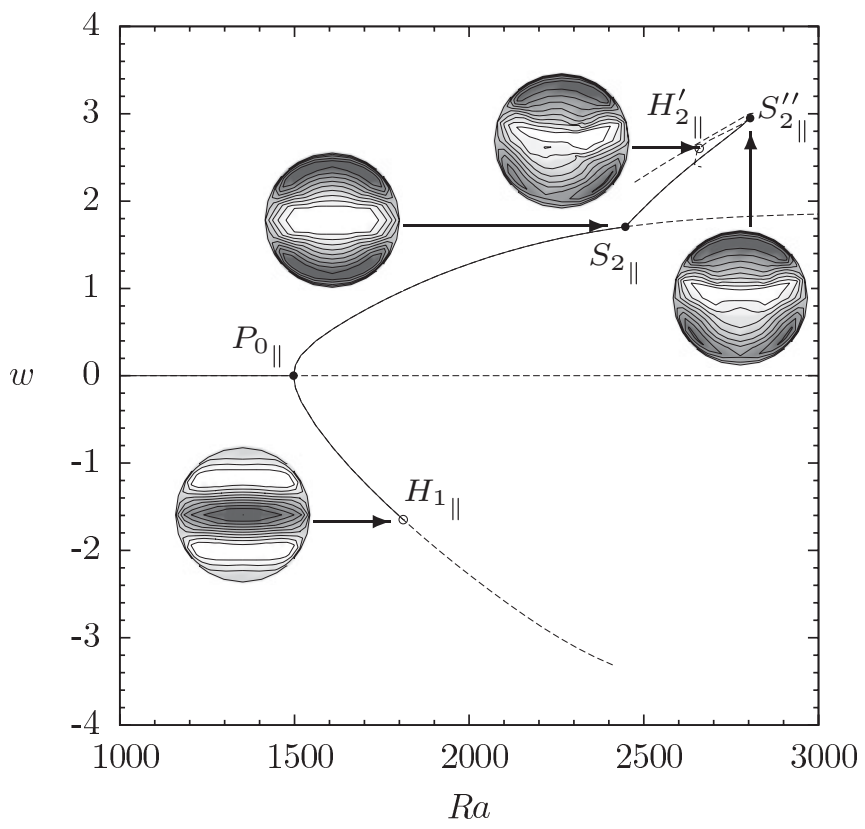

FIG. 15. Bifurcation diagram for a liquid metal $(\operatorname{Pr}=0.0286)$ in a cavity with $A=1.5$ when a horizontal magnetic field is applied $(\mathrm{Ha}=10)$. Note the unfolding of the bifurcation $S_{1}$ obtained for $\mathrm{Ha}=0$ and the change of $S_{2}$ to $S_{2 \|}$. Insets show vertical velocity contours at midheight in the cavity.

perturbation. The new stable branch is eventually destabilized via a Hopf bifurcation at $\mathrm{Ra}_{H_{2}}=2643$.

As shown in Sec. III A, the presence of a horizontal magnetic field will change the primary axisymmetric $m=0$ mode to an $m=0 / 2_{\|}$mode. If the changes of the flow fields remain weak for a small value of $\mathrm{Ha}$ (as $\mathrm{Ha} \leqslant 1$ ), they become more important for sufficiently large values of $\mathrm{Ha}$, as $\mathrm{Ha}=10$. The bifurcation diagram obtained for $\mathrm{Ha}=10$ is shown in Fig. 15. As expected, the primary bifurcation is delayed. It occurs at $\operatorname{Ra}_{P_{0}}=1497$, at a transcritical bifurcation where two distinct branches of $m=0 / 2_{\|}$solutions appear. The supercritical branch remains stable up to a Hopf bifurcation point at $\mathrm{Ra}_{H_{1}}=1811$. The secondary bifurcation obtained for $\mathrm{Ha}=0$ at $\mathrm{Ra}_{S_{1}}$, which changed the symmetry of the solutions from $O(2)$ to $D_{2}$, was to disappear in the presence of a horizontal magnetic field as the symmetry of the problem is already changed to $D_{2}$. But, we can see that, for $\mathrm{Ha}=10$, there even remains no memory of this early bifurcation at $S_{1}$ as the solution directly moves toward the point $H_{1_{\|}}$ without exploring the neighborhood of $S_{1}$. The disconnected branch corresponding to $m=0 / 2_{\perp}$ solutions is unstable. For $\mathrm{Ha}=10$, this branch has moved far away from the stable branch and, as we will see later, it lies outside the Ra range plotted in the figure.

The subcritical branch is also changed by the horizontal magnetic field. This branch, which corresponds to $m=0 / 2_{\|}$ solutions with upflow at the center, remains stable in a larger range of $\mathrm{Ra}$ than for $\mathrm{Ha}=0$, as the point $S_{2}$ is moved up to $S_{2 \|}$ at $\operatorname{Ra}_{S_{2 \|}}=2447$, where a pitchfork bifurcation associated with an $m=3_{\|}$perturbation occurs. Note that the point $S_{2}$ is in fact split to the two points $S_{2_{\|}}$and $S_{2_{\perp}}$ when the horizontal magnetic field is applied, but $\mathrm{Ra}_{S_{2_{\perp}}}$ is already far above $\mathrm{Ra}_{S_{2 \|}}$ 


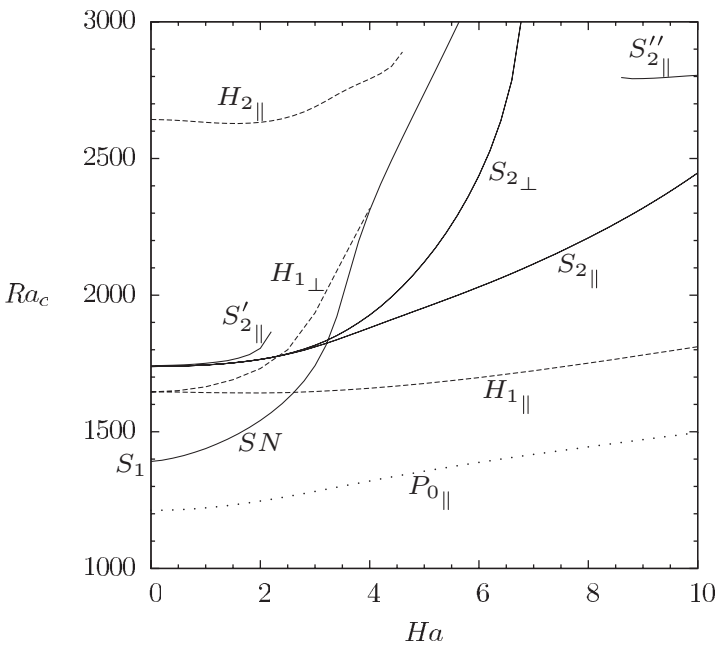

FIG. 16. Paths of the main bifurcation points found in the bifurcation diagrams of Figs. 14 and 15 for $0 \leqslant \mathrm{Ha} \leqslant 10(\mathrm{Pr}=$ $0.0286, A=1.5)$.

for $\mathrm{Ha}=10$. The two new branches initiated at $S_{2_{\|}}$(only one of them is plotted on the bifurcation diagram) correspond to solutions with only one plane of symmetry, the vertical central plane perpendicular to the direction of the imposed magnetic field. The new solutions denoted as $m=0 / 2 / 3_{\|}$solutions are stable up to a steady bifurcation point $S_{2_{\|}}^{\prime \prime}$ at $\mathrm{Ra}_{S_{2_{\|}}^{\prime \prime}}=2804$. The branches then reverse direction at a saddle-node point and remain unstable. The bifurcation at $S_{2 \|}^{\prime \prime}$ is subcritical, so that the branches initiated at this point and that go through a Hopf bifurcation point $H_{2 \|}^{\prime}$ are unstable.

The transition between these two diagrams is depicted in Fig. 16 through the paths of the bifurcation points as a function of Ha. As expected, the primary bifurcation threshold $\mathrm{Ra}_{P_{0_{\|}}}$

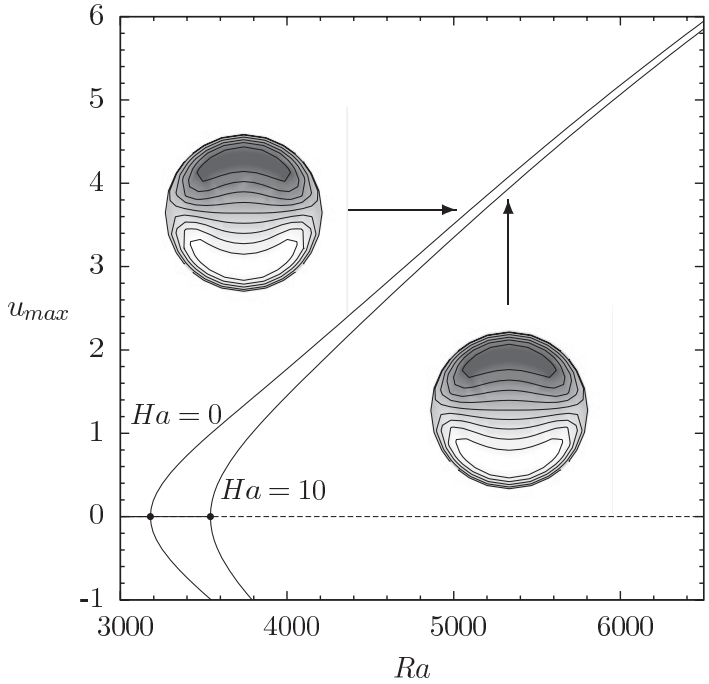

(a) continuously increases for increasing $\mathrm{Ha}$, which corresponds to a delayed onset of the convection. The secondary bifurcation on the supercritical primary branch at $\mathrm{Ra}_{S_{1}}$ disappears as soon as Ha is nonzero. The point $S_{1}$, however, becomes a saddlenode point on a disconnected branch. The path of this point denoted as $S N$ shows a very strong increase with Ha, with $\mathrm{Ra}_{S N}$ already reaching 3000 for a value of Ha smaller than 6 . The Hopf bifurcation that appeared on the secondary branches at $\mathrm{Ra}_{H_{1}}$ for $\mathrm{Ha}=0$ gives two distinct Hopf bifurcation points for $\mathrm{Ha} \neq 0: H_{1_{\|}}$on the supercritical primary branch and $H_{1_{\perp}}$ on the disconnected branch. After a small initial decrease, $R a_{H_{1 \|}}$ is slowly stabilized when Ha is increased, in a similar way as the primary bifurcation at $\operatorname{Ra}_{P_{0}}$. In contrast, $R a_{H_{1}}$ is strongly stabilized, but it quickly disappears by collision with the saddle-node point $S N$. Concerning the subcritical primary branch, the circular pitchfork bifurcation at $\mathrm{Ra}_{S_{2}}$ gives two pitchfork bifurcations at $\mathrm{Ra}_{S_{2 \|}}$ and $\mathrm{Ra}_{S_{2_{\perp}}}$ when the horizontal magnetic field is applied. Both bifurcations are stabilized as $\mathrm{Ha}$ is increased, but beyond $\mathrm{Ha}=3$, the increase of $\mathrm{Ra}_{S_{2}}$ is much stronger than that of $\mathrm{Ra}_{S_{2}}$, and $\mathrm{Ra}_{S_{2}}$ reaches 3000 for a value of Ha smaller than 7. It was not possible to follow the other bifurcation points $S_{2 \|}^{\prime}, H_{2 \|}$, and $S_{2 \|}^{\prime \prime}$ on the whole $\mathrm{Ha}$ range from 0 to 10. $S_{2 \|}^{\prime}$ was obtained only for $\mathrm{Ha} \leqslant 2.2, H_{2 \|}$ for $\mathrm{Ha} \leqslant 4.6$, and $S_{2}^{\prime \prime}$ for $8.6 \leqslant \mathrm{Ha} \leqslant 10$. This means that, for the secondary branches involved in this part of the diagram, there is not a simple transition between the results obtained for $\mathrm{Ha}=0$ and those obtained for $\mathrm{Ha}=10$.

\section{Magnetic field effect on the convection induced at a supercritical primary bifurcation}

We now consider a smaller aspect ratio cavity $(A=0.5)$ for which the first bifurcation is supercritical and associated with an asymmetric $m=1$ mode. The bifurcation diagram without

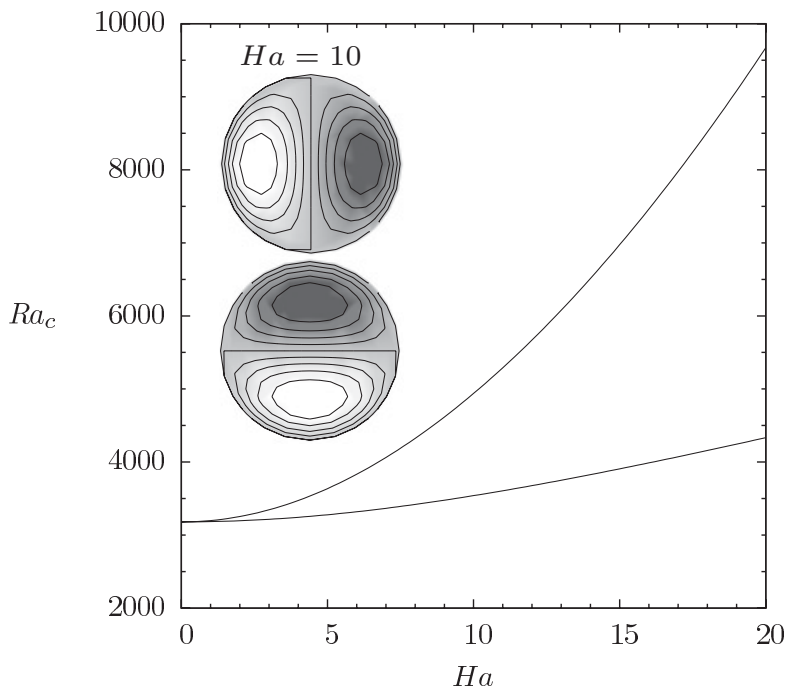

(b)

FIG. 17. (a) Bifurcation diagrams for a liquid metal $(\operatorname{Pr}=0.0286)$ in a cavity with $A=0.5$ in a case without magnetic field (Ha $=0)$ and in a case when a horizontal magnetic field is applied $(\mathrm{Ha}=10)$. The primary bifurcation is either circular pitchfork and associated with an $m=1$ mode $(\mathrm{Ha}=0)$ or pitchfork and associated with an $m=1_{\|}$mode $(\mathrm{Ha}=10)$. (b) Stabilization of the primary bifurcation by the horizontal magnetic field. Two critical curves corresponding to an $m=1_{\|}$mode (weakly stabilized) and an $m=1_{\perp}$ mode (strongly stabilized) are obtained. Insets show vertical velocity contours at midheight in the cavity. 
magnetic field $(\mathrm{Ha}=0)$ for this configuration is plotted in Fig. 17(a) for the same parameter values as in the previous section $(\mathrm{Pr}=0.0286, \mathrm{Bi}=100$, and $\mathrm{Ma}=0)$. The first bifurcation is a circular pitchfork bifurcation, which occurs at $\mathrm{Ra}=3180$ and leads to an asymmetric one-roll flow structure (defined to within a rotation). This flow remains stable in the range of Ra studied ( $\mathrm{Ra} \leqslant 6500)$. As in the previous cases, the solution branches initiated at other primary bifurcation points remain unstable and do not interact with the stable solution.

The stabilization of the first primary bifurcation point as a function of Ha is shown in Fig. 17(b). The initial $m=1$ mode gives two one-roll modes oriented either parallel $\left(m=1_{\|}\right)$or perpendicular $\left(m=1_{\perp}\right)$ to the magnetic field. The $m=1_{\|}$ mode is clearly less stabilized with a relative increase $\Delta \mathrm{Ra}_{\|}=$ 0.36 for Ha varying from 0 to 20 , whereas the $m=1_{\perp}$ mode is strongly stabilized with $\Delta \mathrm{Ra}_{\perp}=2.04$. The $m=1_{\|}$mode will then be the first critical mode with a horizontal magnetic field in this case. The bifurcation diagram obtained when a horizontal magnetic field is applied $(\mathrm{Ha}=10)$ is also shown in Fig. 17(a). The onset of the $m=1_{\|}$flow occurs at Ra $=3540$ at a supercritical pitchfork bifurcation (symmetry breaking from $D_{2}$ to $Z_{2}$ ). The two branches of $m=1_{\|}$states (symmetric from each other) still remain stable in the studied range of Ra. Note, finally, that the flow structure has not been changed much by the horizontal magnetic field, except for the fact that it is now an oriented flow structure.

\section{CONCLUSION}

The effect of a constant horizontal magnetic field on the development of convection in a cylindrical cavity heated from below and with a free surface at the top has been investigated. Such a magnetic field changes the symmetries of the problem from $O(2)$ to $D_{2}$, so that the eigenmodes that determine the convective flow at onset are modified. The axisymmetric mode is changed to an asymmetric $m=0 / 2$ mode, whereas the other asymmetric modes that were multiply defined (to within a rotation) have now only two possible orientations connected to the direction of the applied magnetic field. The three main modes ( $m=0,1$, and 2$)$ then give five modes: two $m=1$ modes $\left(m=1_{\|}\right.$and $\left.m=1_{\perp}\right)$, two $m=0 / 2 \operatorname{modes}\left(m=0 / 2_{\|}\right.$ and $\left.m=0 / 2_{\perp}\right)$, and an $m=2$ mode $\left(m=2^{a}\right)$.

The effect of the magnetic field on the onset of convection has been studied by following the paths of the primary bifurcation points (expressed as $\mathrm{Ra}_{c}$ ) as a function of the Hartmann number Ha. It was found that the modes are differently stabilized, weakly if the axis of the rolls is parallel to $B\left(m=1_{\|}\right.$ and $\left.0 / 2_{\|}\right)$, strongly if the axis of the rolls is perpendicular to $B\left(m=1_{\perp}\right.$ and $\left.0 / 2_{\perp}\right)$, and moderately for the $m=2^{a}$ mode. These stabilizations are mainly due to the stabilizing energy contribution coming from the Lorentz force, but the change of the flow structure also gives a contribution to the increase of $\mathrm{Ra}_{c}$ through the evolution of the buoyant force. The variation of the primary thresholds with the aspect ratio of the cavity has also been studied in a case with magnetic field $(\mathrm{Ha}=3)$. The more striking result is the interaction between the modes $m=0$ and 2. When the magnetic field is applied, these modes can not intersect any more. Moreover, the weakly stabilized $m=0 / 2_{\|}$mode comes from either the $m=0$ mode or the $m=2$ mode, more precisely, from the one of the two modes that has the smallest threshold for the chosen aspect ratio.

The nonlinear evolution of the convection in the presence of the horizontal magnetic field has then been studied for two values of the Prandtl number: $\operatorname{Pr}=1$ and $\operatorname{Pr}=0.0286$. For $\operatorname{Pr}=1$, the study was focused on a cavity with aspect ratio $A=1.5$, a case where a complex dynamics with strongly subcritical branches was obtained without magnetic field. The changes induced by the magnetic field were analyzed for $\mathrm{Ha}=$ 1. Convection sets in as an $m=0 / 2_{\|}$mode so that the former secondary subcritical bifurcation to such a mode disappears, giving an imperfect bifurcation, which has been characterized and modeled. Moreover, the transitions to $m=0 / 1$ solutions are split into two distinct transitions to $m=0 / 1_{\|}$and $0 / 1_{\perp}$ solutions, which evolve distinctively but in a similar way. For $\mathrm{Pr}=0.0286$, a value corresponding to liquid metals, the effect of the magnetic field was studied for two aspect ratios $A=$ 1.5 and 0.5 and for $\mathrm{Ha}=10$. For this Prandtl number, the dynamical evolutions are a little more simple. For $A=1.5$, the onset of convection as an $m=0 / 2_{\|}$mode when the magnetic field is applied still induces the disappearance of the secondary bifurcation, which was, however, supercritical in this case. For $A=0.5$, the main change is that the stable $m=1$ flow is replaced by a slightly delayed stable $m=1_{\|}$flow.

\section{ACKNOWLEDGMENTS}

This work was granted access to the HPC resources of IDRIS under the allocations 2008-021559, 2009-021559, 2010-021559, and 2011-021559 made by GENCI (Grand Equipement National de Calcul Intensif).
[1] H. Ben Hadid, D. Henry, and S. Kaddeche, J. Fluid Mech. 333, 23 (1997).

[2] H. Ben Hadid and D. Henry, J. Fluid Mech. 333, 57 (1997).

[3] H. Ben Hadid and D. Henry, J. Cryst. Growth 166, 436 (1996).

[4] T. Alboussière, J. P. Garandet, and R. Moreau, J. Fluid Mech. 253, 545 (1993).

[5] D. Henry, A. Juel, H. Ben Hadid, and S. Kaddeche, Phys. Fluids 20, 034103 (2008).

[6] S. Chandrasekhar, Hydrodynamic and Hydromagnetic Stability (Clarendon, Oxford, 1961).

[7] C. Benzid, S. Kaddeche, A. Abdennadher, D. Henry, and H. Ben Hadid, C. R. Mec. 337, 291 (2009).
[8] R. Touihri, H. Ben Hadid, and D. Henry, Phys. Fluids 11, 2089 (1999).

[9] B. C. Houchens, L. Martin Witkowski, and J. S. Walker, J. Fluid Mech. 469, 189 (2002).

[10] J. S. Walker, D. Henry, and H. Ben Hadid, J. Cryst. Growth 243, 108 (2002).

[11] J. S. Vrentas, R. Narayanan, and S. S. Agrawal, Int. J. Heat Mass Transfer 24, 1513 (1981).

[12] P. C. Dauby and G. Lebon, J. Fluid Mech. 329, 25 (1996).

[13] R. Touihri, A. El Gallaf, H. Ben Hadid, and D. Henry, Phys. Rev. E 84, 056302 (2011).

[14] R. Moreau, Magnetohydrodynamics (Kluwer Academic, Dordrecht, 1990). 